\title{
APPLICATIONS OF GROUND PENETRATING RADAR IN GEOTECHNICAL INVESTIGATION
}

by

\section{DAMIAN MOODIE}

Bachelor of Engineering in Construction Engineering University of Technology, Kingston, Jamaica, 2007

a master's research project

presented to Ryerson University

in partial fulfillment of the

requirements for the degree of

Master of Engineering

in the Program of

Civil Engineering

Toronto, Ontario, Canada, 2017

C DAMIAN MOODIE, 2017 


\section{Author's Declaration}

I hereby declare that I am the sole author of this research project. This is a true copy of the research project, including any required final revisions.

I authorize Ryerson University to lend this document to other institutions or individuals for the purposes of scholarly research.

I further authorize Ryerson University to reproduce this research project by photocopying or by

other means, in total or in part, at the request of other institutions or individuals for the purposes of scholarly research.

I understand that my research project may be made electronically available to the public. 


\title{
APPLICATIONS OF GROUND PENETRATING RADAR IN GEOTECHNICAL INVESTIGATION
}

Master of Engineering, 2017

DAMIAN MOODIE

Civil Engineering Program

Ryerson University

\begin{abstract}
There are clearly risks and a fair degree of uncertainties involved in geotechnical investigation for the reason that only limited boreholes can be used in projects, due to budget restraints. These risks are further increased or decreased subject to the geotechnical engineers' experiences and judgments.

Ground Penetrating Radar (GPR) is a geophysical technique that provides continuous nondestructive soil profiling from the surface or from inside a borehole by sending, receiving and averaging multiple radio wave pulses into the subsurface at centimeter increments $(\mathrm{cm}) \mathrm{scale}$ normally ranging between $0.5 \mathrm{~cm}$ to $1 \mathrm{~cm}$ step size.

This project focuses on the principles, procedures, applications and limitations of GPR use in geotechnical exploration. To evaluate its potentials for reducing risk and uncertainties associated with soil profile presumptions between boreholes, also to evaluate if GPR can provide objective quantifiable data that can be understood by any level of geotechnical engineers.
\end{abstract}




\section{Acknowledgements}

The author would like to thank and express his deep appreciation to his advisor Dr. Jinyuan Liu, from Ryerson University for his help, patience and continuous support throughout the development of this research project. Dr. Liu devoted his time and efforts to helping with the completion of this research project. His supervision and advice were very valuable and are greatly appreciated.

The author would like to thank Mr. Courtney Simons from WCI Geophysics in Jamaica, who permitted the author to observe field data collection and to use software during post data processing, during their investigation for a hotel expansion project. His assistance and input are very much appreciated.

The author is very grateful to his family, friends, and colleagues for their great support and the continued encouragement provided, to successfully complete this study. 


\section{Table of Contents}

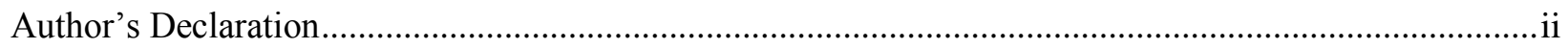

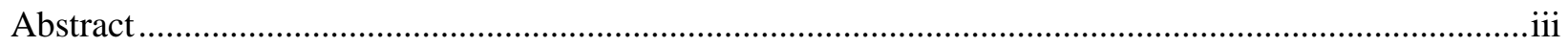

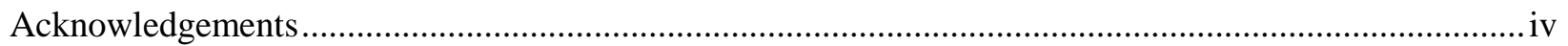

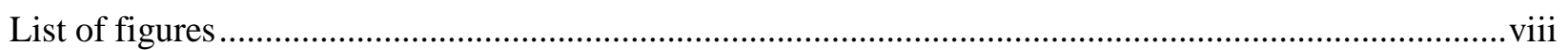

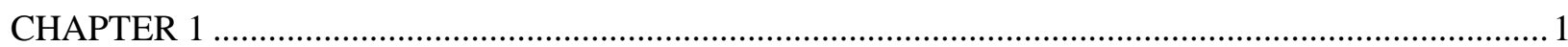

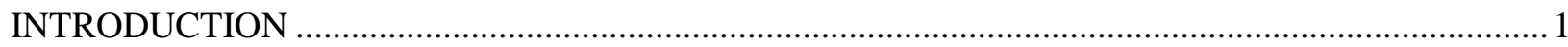

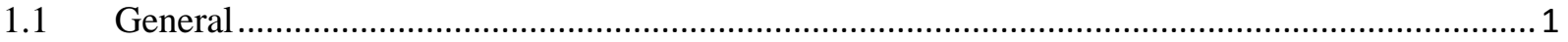

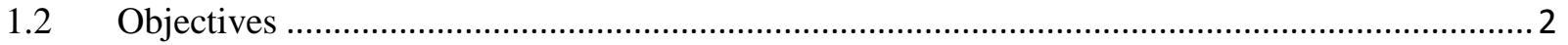

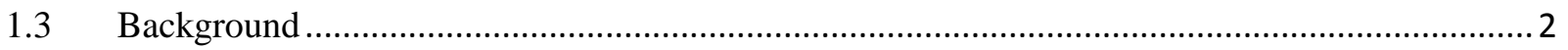

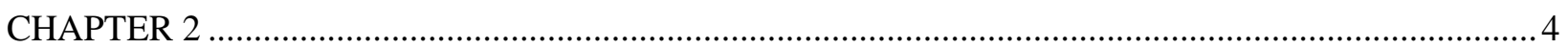

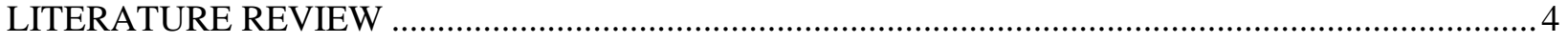

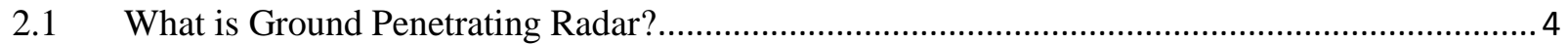

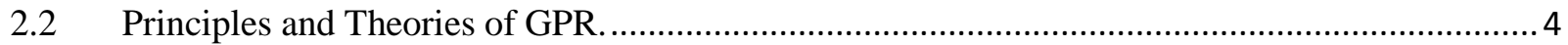

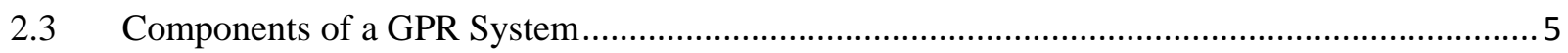

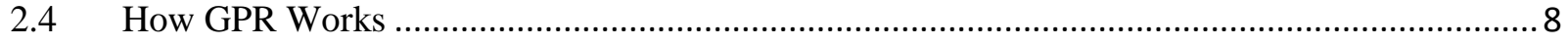

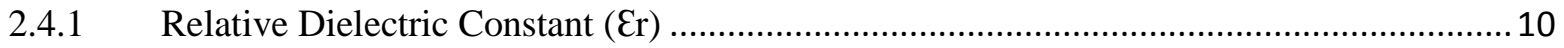

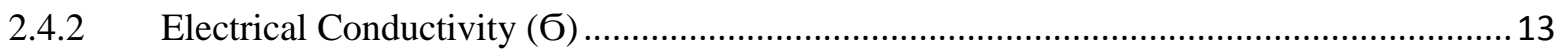

2.4.3 Obtaining Depth from Time Window ....................................................................... 14

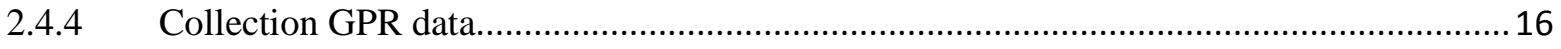

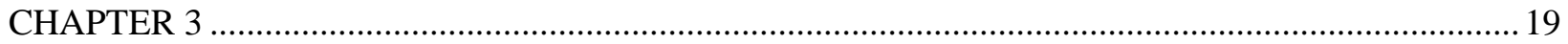

APPLICATIONS IN GEOTECHNCAL ENGINEERING …........................................................... 19

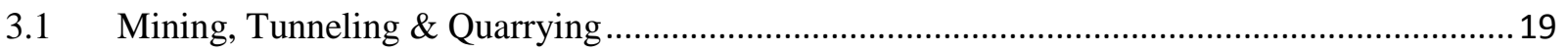

3.2 Ground Water Table, Karst Evaluation, and Soil Stratification.............................................. 19

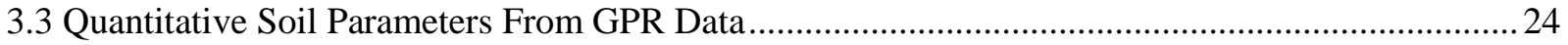

3.3.1 Ground Penetrating Radar used to Detect Changes in Void Ratio Before and After Liquefaction

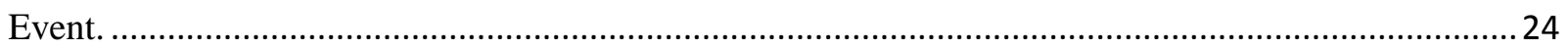

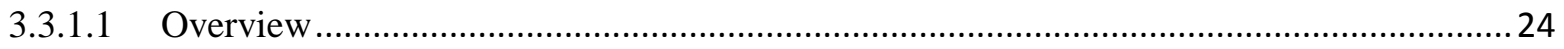

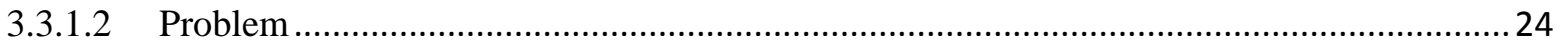

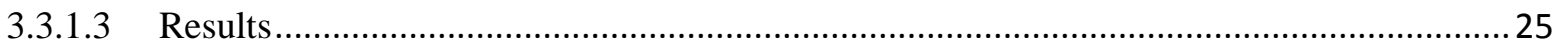

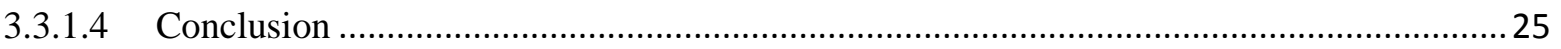

3.3.2 Ground Penetrating Radar used to Determine Soil Water Content............................................. 26 


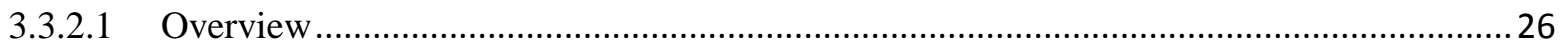

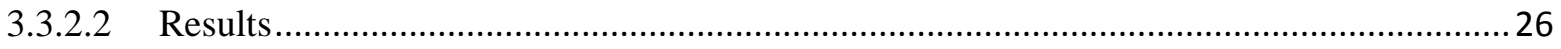

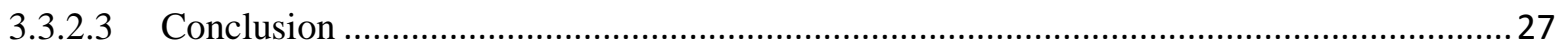

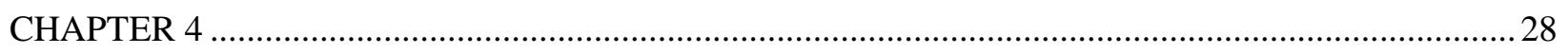

OBSERVATIONS, ANALYSIS AND REPORT FOR HOTEL EXPANSION, JAMAICA ....................28

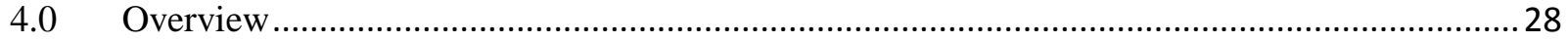

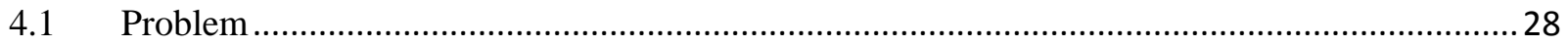

4.2 Background Information Prior to GPR Surveys …............................................................. 29

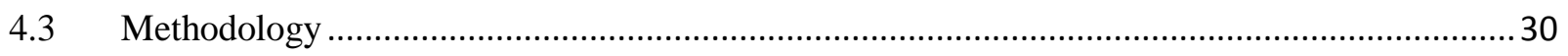

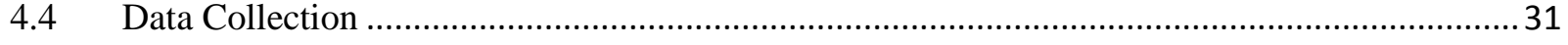

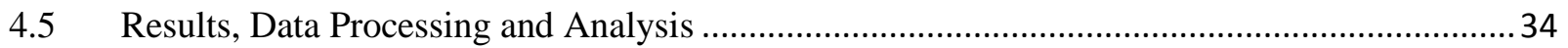

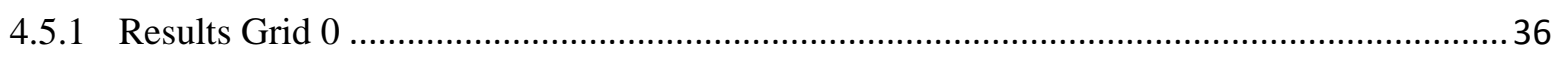

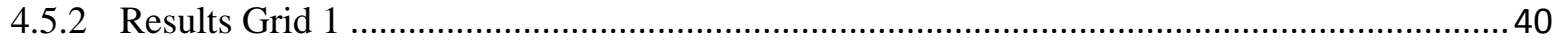

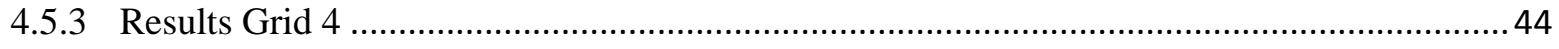

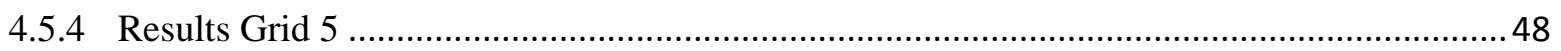

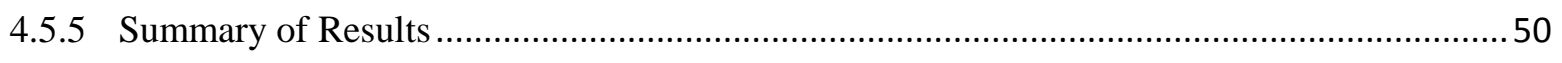

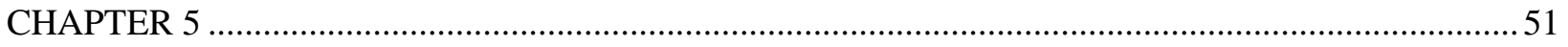

CONCLUSION, RECOMMEDATION AND LIMITATIONS …...................................................... 51

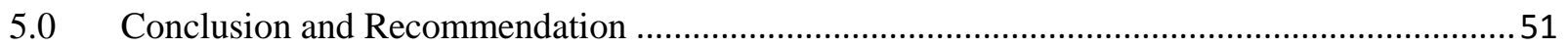

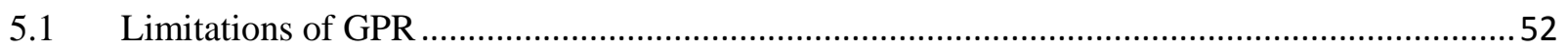

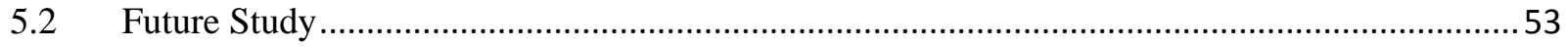

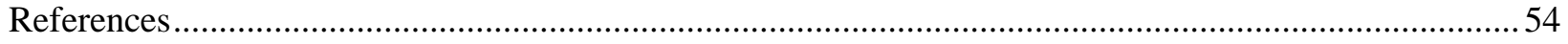




\section{List of figures}

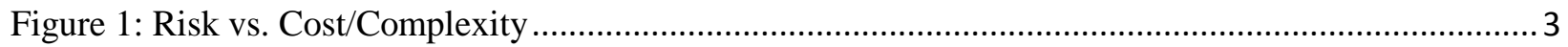

Figure 2: Electromagnetic Spectrum with GPR Band Range. .......................................................... 5

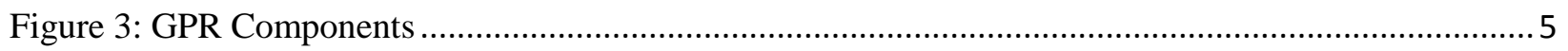

Figure 4: Power Transformation Cycle from Tx to Rx....................................................................... 6

Figure 5: GPR penetration depth range of different material ........................................................... 8

Figure 6: Conical scattering from target reflection ....................................................................................

Figure 7: Frequency vs. Attenuation for EM radio waves .................................................................. 9

Figure 8: Principle of GPR reflection at the boundary ...................................................................... 11

Figure 9: How GPR signals are transmitted and received at the boundaries. ......................................... 11

Figure 10: GPR data with time and depth on vertical axis ................................................................. 14

Figure 11: Constant antenna offset reflection profiling .................................................................... 17

Figure 12: Transmission mode or Wide Angle Reflection and Refraction (WARR) ...............................17

Figure 13: Methods of borehole GPR data collection....................................................................... 18

Figure 14: Data collected from a massive granite at 70m depth - black reflections are fractures.............19

Figure 15: Detection of a karstic cave formed in limestone by post data processing (a), this cave was

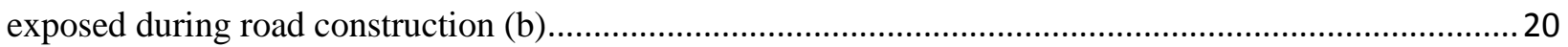

Figure 16: Dune 1, Ground Penetrating Radar Reflections (a), Interpretation of reflections (b)...............21

Figure 17: Dune 2, Ground Penetrating Radar reflections (a), Interpretation of reflections (b) .................21

Figure 18: Soil stratigraphic profile and water table interface .............................................................. 22

Figure 19: Sub-Bottom Stratigraphy Image in fresh water ................................................................. 23

Figure 20: Results showing GPR predicted settlement vs. observed settlement. ......................................25

Figure 21: Comparison between GPR and CTP soil water content. ................................................... 26

Figure 22: Showing site layout, existing structures and equipment onsite during data collection. ............31

Figure 23: Noggin Smart Cart GPR machine 100MHz antenna during data collection ............................ 33

Figure 24: Schematic Illustration of Building A GPR Grid Layout ...................................................... 35

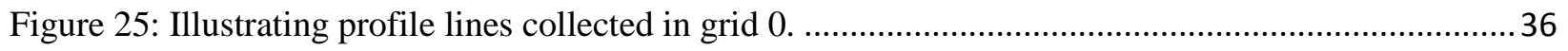

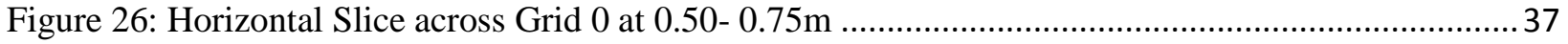

Figure 27: Processed vertical section of line 17 profile line indicating slice lines at $1.50-2.00 \mathrm{~m} \ldots \ldots \ldots \ldots . . . .38$

Figure 28: Vertical section of line 23 profile line indicating slice lines at $0.75-1.00 \mathrm{~m}$........................... 39

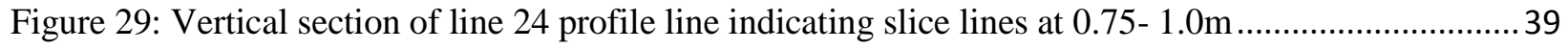




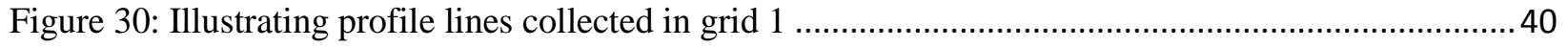

Figure 31: Horizontal Slice across Grid 1 at $0.50-0.75 \mathrm{~m}$ depth.......................................................... 41

Figure 32: Vertical section of profile line 16 with slice lines at 1.50 to $1.75 \mathrm{~m}$...................................... 42

Figure 33: Vertical section of profile line 14 with slice lines at 0.25 to $0.50 \mathrm{~m}$................................... 42

Figure 34: Section of profile collected during random walk through ................................................. 43

Figure 35: Illustrating profile lines collected in grid 4 ....................................................................... 44

Figure 36: Horizontal Slice across Grid 4 at 0.50-0.75m depth........................................................... 45

Figure 37: Horizontal Slice across Grid 4 at 1.25- 1.50m depth........................................................ 46

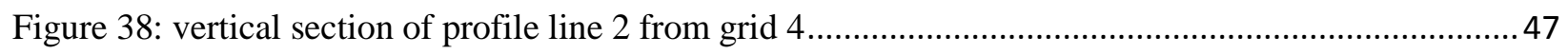

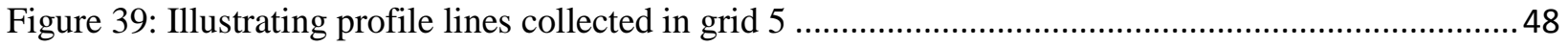

Figure 40: Horizontal slice across Grid 4 at 3.25- 3.50m depth ......................................................... 49

Figure 41: Schematic illustration of results across the site .................................................................. 50

\section{List of tables}

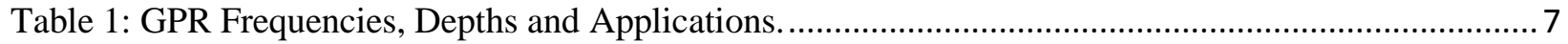

Table 2: Relative Dielectric Constants and Velocity of Various Soils. .................................................. 12

Table 3: Calculated expected contrast intensity at boundaries. ..............................................................29 


\section{CHAPTER 1 \\ INTRODUCTION}

\subsection{General}

Planning a geotechnical investigation to acquire satisfactory data for design while controlling cost can be a challenging task. The number of boreholes, spacing, and locations depend on a number of factors, including but not limited to cost, location, and type of structure to be constructed, as well as the site's conditions. A geotechnical engineer is compelled to deduce a soil profile, estimate strength and the settlement characteristics of the site by interpolating between borings made at $15 \mathrm{~m}$ or greater (Terzaghi, 1996).

With the above in mind, there is vast leeways for errors and misjudgments of the subsurface soils. Hence the reliability of the design and computed engineering properties depend on the differences between real a deduced soil profile and properties. If significant subsoil feature should escape the attention of the design team, the design may be unsatisfactory or lead to future failure.

According to (Terzaghi, 1996), experience has shown that misjudgment of subsoil conditions may be placed in three categories:

1. Excessive soil disturbance may influence test results due to significant difference between test and field conditions.

2. Failure to detect the most unfavorable subsoil condition compatible with the field data.

3. Inadequate communication between designers and contractors, resulting in failure to detect significant deviation from design specification and field procedures.

Interpolating to deduce the soil profile between boreholes is highly subjective and results will dependent on the geotechnical engineer's judgments and experiences. That is to say, for the same borelog data, a junior engineer may provide different soil profile results when compared with the results of a senior geotechnical engineer. To minimize (and possibly eliminate) the risks of soil and design failure due to unforeseen subsurface event, a method of constant soil profiling would 
provide conclusive soil data and subsoil stratigraphy, along with contouring of the various layers at varying depths.

\section{$1.2 \quad$ Objectives}

This project report seeks to determine the extent to which Ground Penetrating Radar (GPR) can be applied to geotechnical investigation to minimize unforeseen risks and uncertainties associated with the un-sampled areas of traditional geotechnical investigation methods. This project will also seek to determine whether geotechnical parameters can be extracted from GPR collected data.

\subsection{Background}

The main purpose for a geotechnical investigation is to determine the soil conditions at a proposed project site for design risk assessment and design purposes. According to (Terzaghi, 1996) a background study is normally conducted before conducting the actual data collection process of the investigation. This background study involves reviewing the site geology, topographic maps, and general conditions of the site as they relates to the accessibility of equipment and required clearance.

Based on background gathering information, some assumptions can be made about the expected soil to be encountered during the later investigation. The geotechnical exploration and data gathering are conducted to validate and refine assumptions made about the likely subsurface conditions. Hence, the exploration becomes an exercise in reducing both the risks and uncertainties related to the amount of information gathered. More information should provide a better understanding of the sub-surface, however the exploration procedure is normally limited by the costs associated therewith. Figure 1 illustrates the increase in cost as technical systems improves, which includes design, materials, inspection, and redundancy measures. The risks can either be decreased to as low as practically achievable ALAPA or as low as reasonably achievable ALARA. 
Several field and laboratory testing procedures are conducted and the results interpreted by a specialist in the field, a geotechnical engineer. A typical geotechnical report should provide (at minimum) the following information:

1. Stratigraphic soil profiles (including depth, spread, and composition of critical soil strata).

2. Ground-water levels

3. Depths of the bed rock

4. Engineering properties (physical, mechanical and sometime chemical properties of the soil).

5. Settlement assessments for the soil

6. Bearing capacity of the soil.

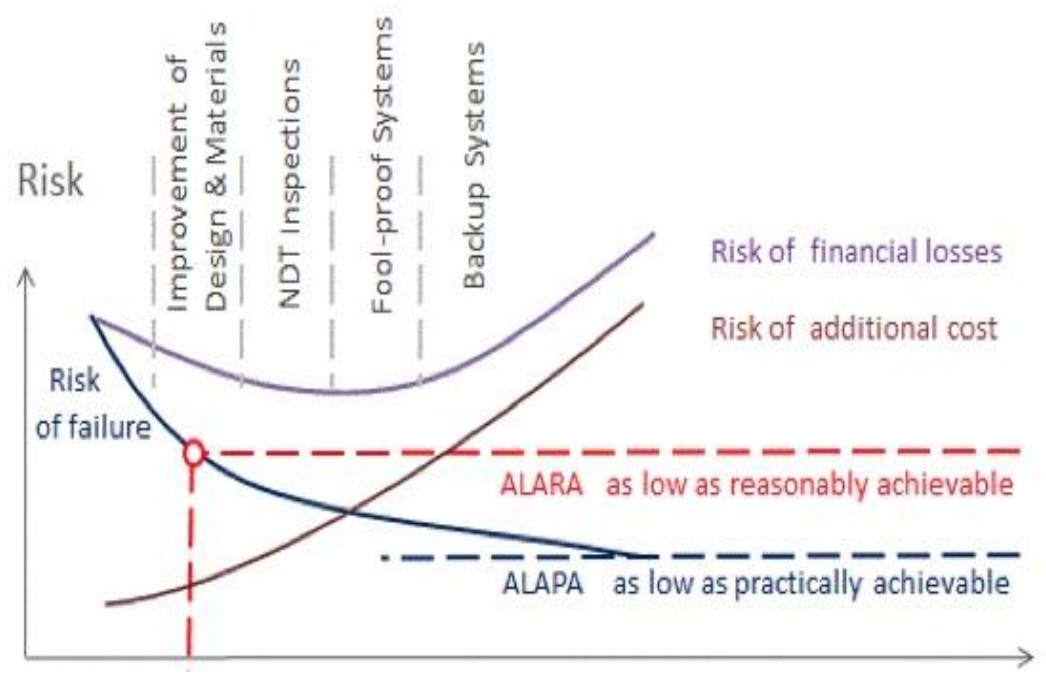

Figure 1: Risk vs. Cost/Complexity 


\section{CHAPTER 2 \\ LITERATURE REVIEW}

\subsection{What is Ground Penetrating Radar?}

Ground Penetrating Radar (GPR) is the common term applied to a method in which radio waves, (typically in the 10 to $1000 \mathrm{MHz}$ frequency range) are used to map features below the surface of natural and man-made structures. Traditionally, between 1960s and 1970s, GPR was mainly focused on mapping structures in the natural ground. However, over the past two decades, major improvements have been achieved in non-destructive testing (NDT) procedures and applications. This is due to developments in data processing and advanced computer systems and/or programs (Annan, 2001). On the forefront of these improved NDT techniques is GPR, which has found wide spread applicability in civil engineering, archeology, and forensic science.

\subsection{Principles and Theories of GPR.}

GPR is a fairly new geophysical technique that has seen major advances over the last two decades thanks to improvements in mass data processing and computer technology, coupled with a better understanding of the requirements and limitations of GPR surveys. There is now an overall sense that the GPR technology has reached a level of maturity.

GPR is a non-destructive, noninvasive technique that uses electromagnetic energy from the radio waves region of the electromagnetic spectrum illustrated in Figure 2 to detect changes and/or variations in dielectric properties of materials or mediums being scanned. GPR has found widespread applicability in geological surveys, geotechnical surveys, pavement assessments, concrete assessments, archeology, forensic investigations and environmental assessments. (Annan, 2001) In these mediums the electromagnetic fields can penetrate to reasonable depths before being absorbed. With GPR, the electromagnetic fields propagate as essentially nondispersive waves. The signal emitted travels through the material, scattered and/or reflected by changes in impedance. The GPR transmitter emits a radio waves signal in all directions, the 
signal penetrates the scanned ground to hit different objects, which have different permittivities due to diverse material compositions.

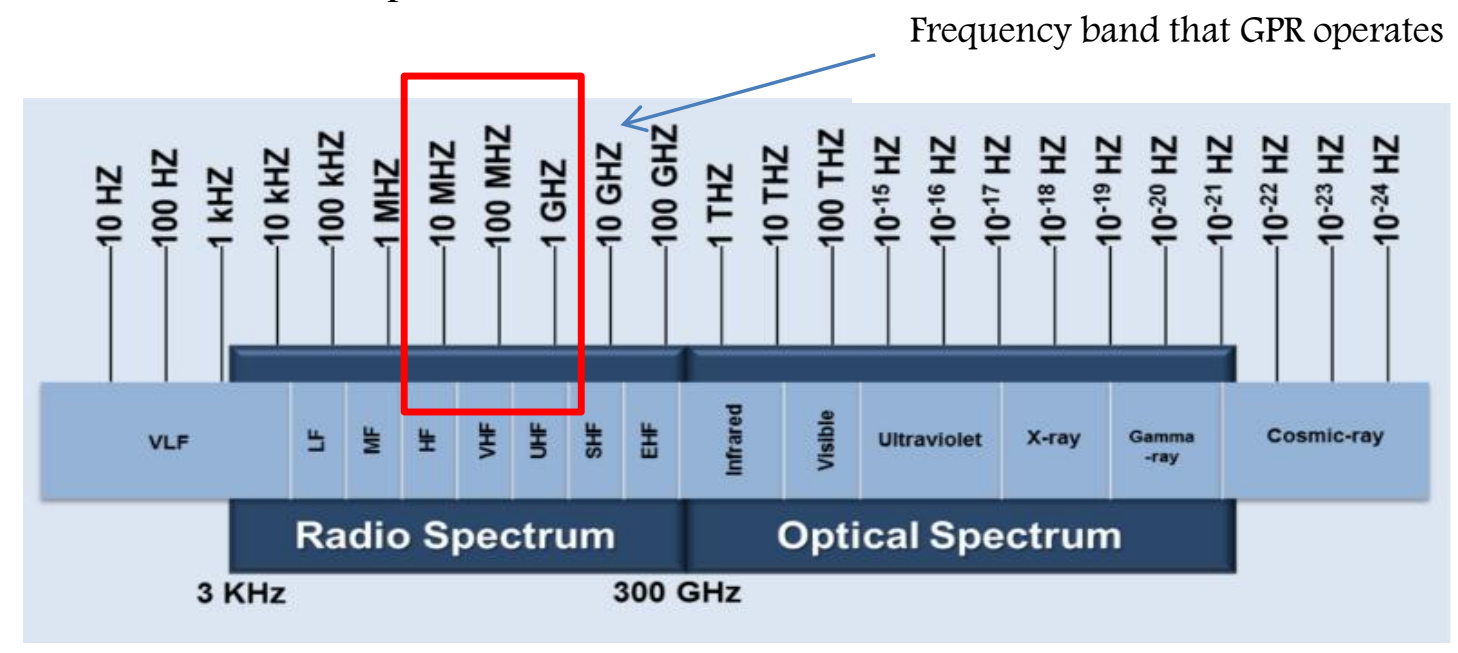

Figure 2: Electromagnetic Spectrum with GPR Band Range.

(NASA, 2012)

\subsection{Components of a GPR System}

A typical GPR System consists of three main components. As illustrated in Figure 3: a control unit (: used to record, store and display reflected signals and position in real time), a power supply system, (normally consisting of a 12 volt battery pack) and antennas (consisting of transmitter $(\mathrm{Tx})$ and receiver $(\mathrm{Rx}))$.

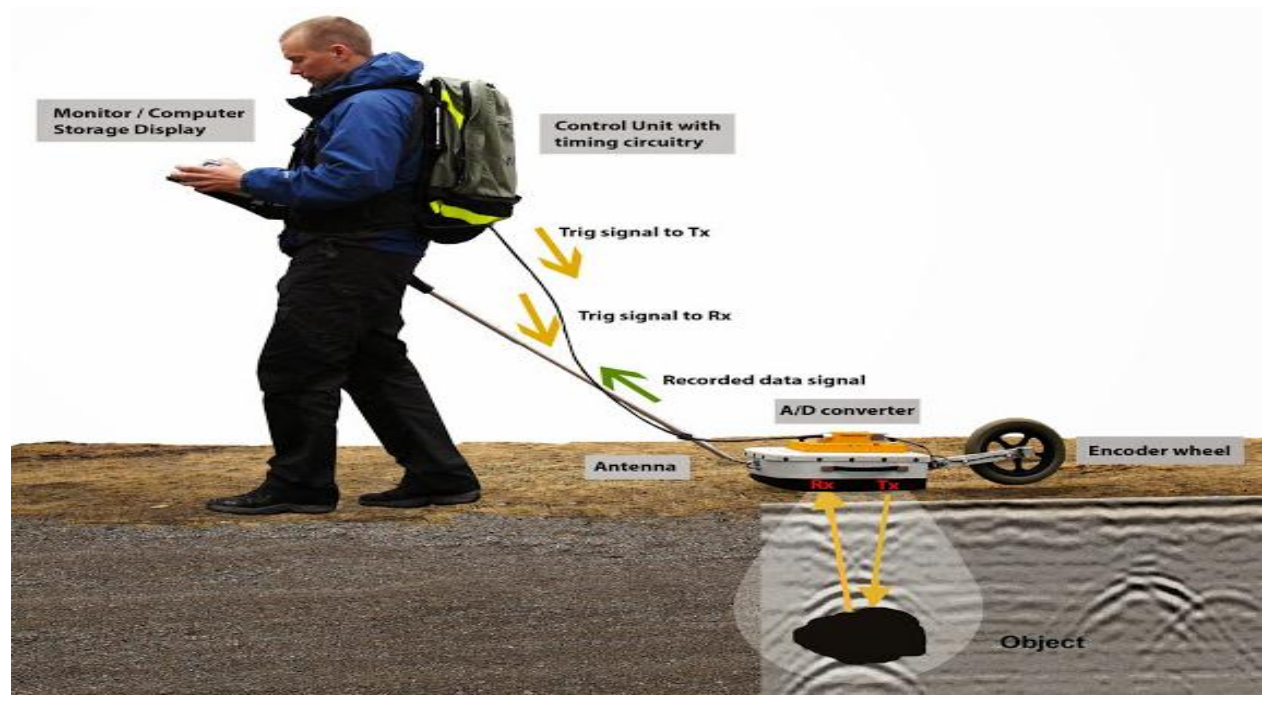

Figure 3: GPR Components

(Jangda, 2014) 


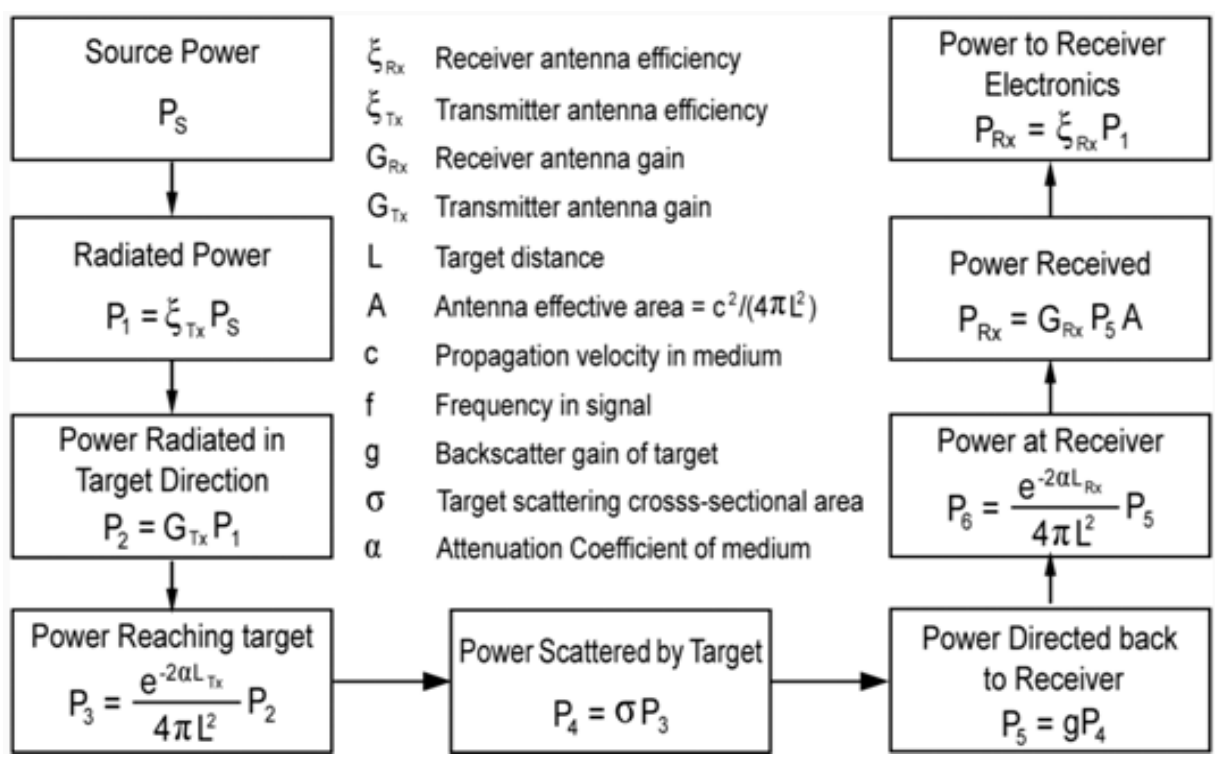

Figure 4: Power Transformation Cycle from Tx to Rx.

(Sensors and Software, 2014)

The control unit consists of electrical components used to trigger multiple short pulses of radar energy into the subsurface by the transmitting antenna, and collecting, storing and displaying the reflected energy from the receiving antenna. Antennas frequency ranges from 10 to $1000 \mathrm{MHz}$ for GPR systems, a single GPR system can be configured to be used with different frequency antenna. Application, size of target, and depth of target, all play an important role in selecting the correct frequency antenna.

Antenna frequency is a very important factor in GPR surveying. The functions thereof are to find a balance between image resolution and depth to the required target for the specific application. Higher frequency antennas like $500-1000 \mathrm{MHz}$ can penetrate to a shallow depth of 1 to $3 \mathrm{ft}$ and are used for (concrete scanning and pavement evaluation). This can produce very clear and accurate resolution of target features. As such, lower frequency antennas like 50-200 MHz can penetrate to very deep targets with lower resolutions are used for mining and both geological and geotechnical investigation. Table 1 shows, typical GPR frequencies, depths and applications for various antenna frequencies. 
Table 1: GPR Frequencies, Depths and Applications.

\begin{tabular}{|c|c|c|c|}
\hline Appropriate Application & $\begin{array}{l}\text { Primary } \\
\text { Antenna } \\
\text { Choice }\end{array}$ & $\begin{array}{l}\text { Secondary } \\
\text { Antenna } \\
\text { Choice }\end{array}$ & $\begin{array}{l}\text { Depth Range } \\
\text { (Approximate) }\end{array}$ \\
\hline $\begin{array}{l}\text { Structural Concrete, } \\
\text { Roadways, Bridge Decks }\end{array}$ & $2600 \mathrm{MHz}$ & $1600 \mathrm{MHz}$ & $0-0.3 \mathrm{~m}(0-1.0 \mathrm{ft})$ \\
\hline $\begin{array}{l}\text { Structural Concrete, } \\
\text { Roadways, Bridge Decks }\end{array}$ & $1600 \mathrm{MHz}$ & $1000 \mathrm{MHz}$ & $0-0.45 \mathrm{~m}(0-1.5 \mathrm{ft})$ \\
\hline $\begin{array}{l}\text { Structural Concrete, } \\
\text { Roadways, Bridge Decks }\end{array}$ & $1000 \mathrm{MHz}$ & $900 \mathrm{MHz}$ & $0-0.6 \mathrm{~m}(0-2.0 \mathrm{ft})$ \\
\hline $\begin{array}{l}\text { Concrete, Shallow Soils, } \\
\text { Archaeology }\end{array}$ & $900 \mathrm{MHz}$ & $400 \mathrm{MHz}$ & $0-1 \mathrm{~m}(0-3 \mathrm{ft})$ \\
\hline $\begin{array}{l}\text { Shallow Geology, Utilities, } \\
\text { UST's, Archaeology }\end{array}$ & $400 \mathrm{MHz}$ & $270 \mathrm{MHz}$ & $0-4 \mathrm{~m}(0-12 \mathrm{ft})$ \\
\hline $\begin{array}{l}\text { Geology, Environmental, } \\
\text { Utility, Archaeology }\end{array}$ & $270 \mathrm{MHz}$ & $200 \mathrm{MHz}$ & $0-5.5 \mathrm{~m}(0-18 \mathrm{ft})$ \\
\hline $\begin{array}{l}\text { Geology, Environmental, } \\
\text { Utility, Archaeology }\end{array}$ & $200 \mathrm{MHz}$ & $100 \mathrm{MHz}$ & $0-9 \mathrm{~m}(0-30 \mathrm{ft})$ \\
\hline Geologic Profiling & $100 \mathrm{MHz}$ & $\begin{array}{l}\text { MLF }(16-80 \\
M H z)\end{array}$ & $0-30 \mathrm{~m}(0-90 \mathrm{ft})$ \\
\hline Geologic Profiling & $\begin{array}{l}\text { MLF }(16-80 \\
M H z)\end{array}$ & None & $\begin{array}{l}\text { Greater than } 30 \mathrm{~m} \\
(90 \mathrm{ft})\end{array}$ \\
\hline
\end{tabular}

Shallow depth geotechnical investigation (High resolution imaging) i.e. small sinkhole, erosion

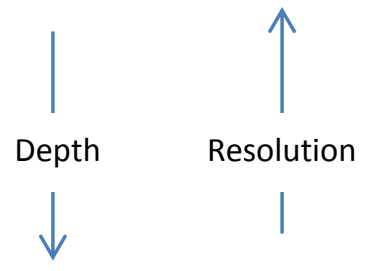

Deep geotechnical investigation less resolution therefore needs larger targets i.e. Bedrock, larger cavity, deep water table 


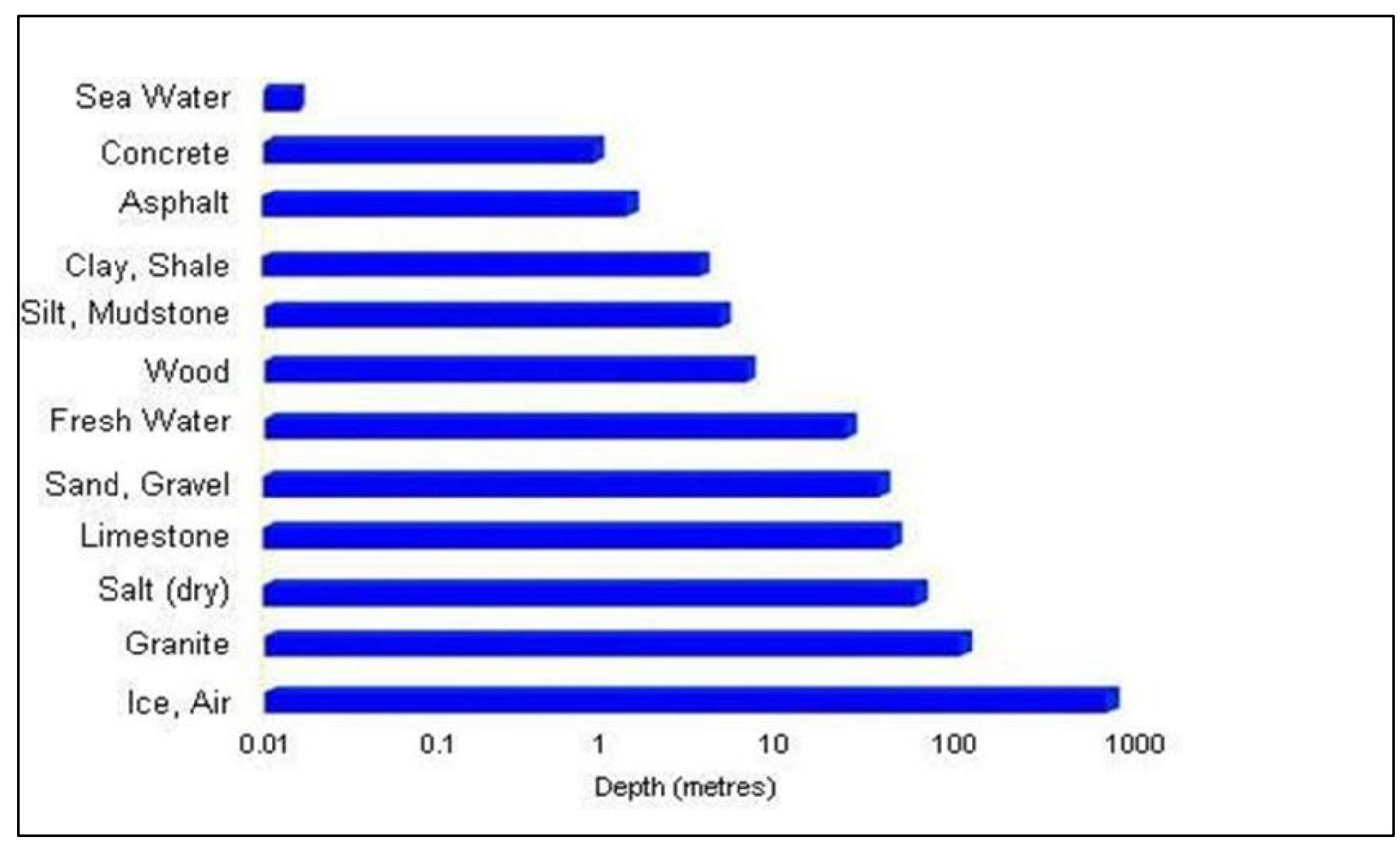

Figure 5: GPR penetration depth range of different material

(Sensors and Software, 2014)

\section{$2.4 \quad$ How GPR Works}

The GPR control unit generates small trigger pulses, which are sent (via the control cables) to the transmitting antenna. At the antenna, the trigger pulses are transformed to a bi-polar transmit pulses which are then transmitted in to the subsurface, while the GPR unit is pulled along the surface. The energy radiates into the subsurface in a conical pattern, as can be seen in Figure 6. Different materials, of varying relative dielectric properties, are encountered as the transmitted pulses radiates into the ground. At these material interface boundaries, of contrasting dielectric material, some of the energy is reflected and detected by the receiving antenna. The received signals are then sent back to the control unit, where they are processed and displayed in realtime.

GPR normally operates within a frequency range of 10 to $1000 \mathrm{MHz}$. Frequencies lower than $1 \mathrm{MHz}$ cause the radio wave to disperse and will not attenuate through the scanned medium, while frequencies higher than $1000 \mathrm{MHz}$, will attenuate at a very fast rate and will be absorbed, causing low penetration and reflection. Figure 7 Illustrate the frequency range that GPR systems normally operate. (Sensors and Software, 2014). 


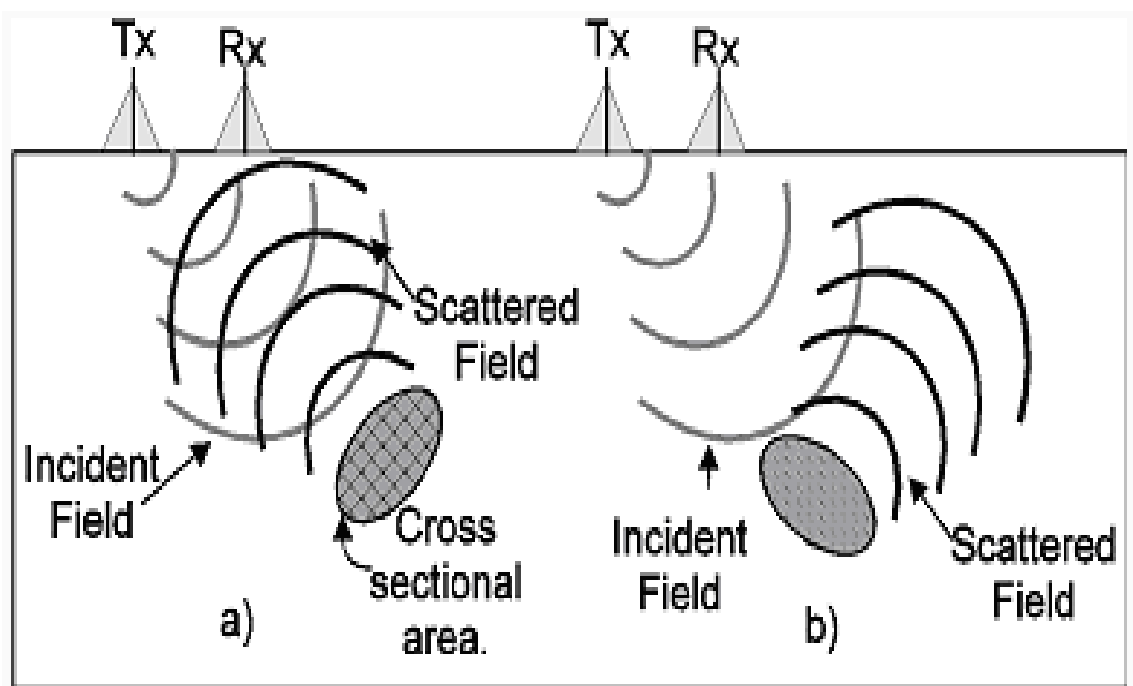

Figure 6: Conical scattering from target reflection

(Anna, 2003)

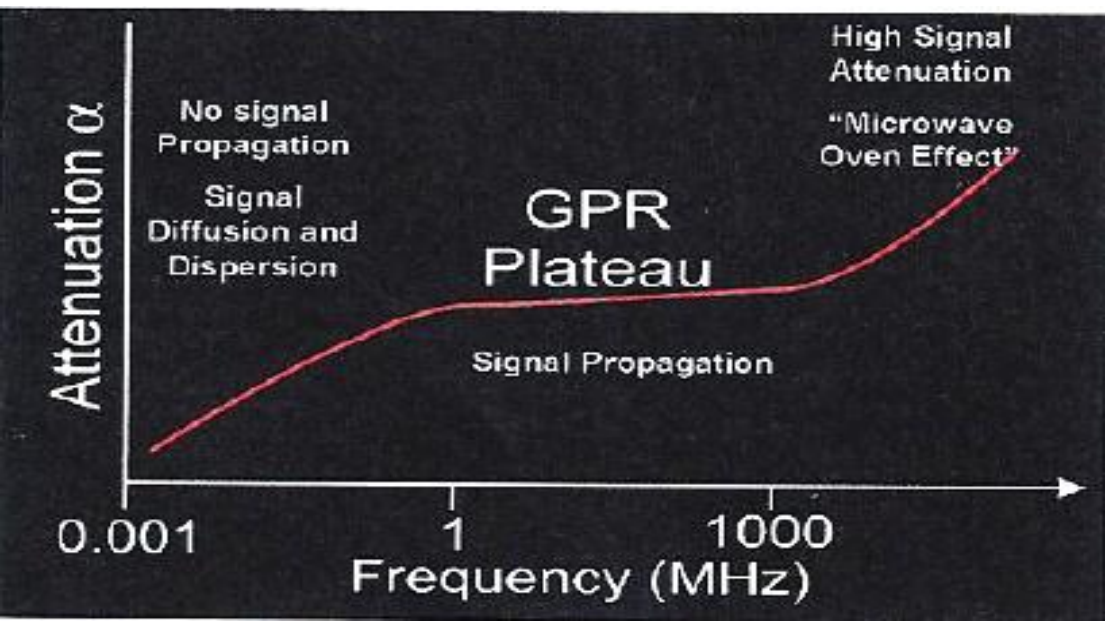

Figure 7: Frequency vs. Attenuation for EM radio waves

(Sensors and Software, 2014) 


\subsubsection{Relative Dielectric Constant $(\mathcal{E r})$}

The velocity and time that the radar pulse takes (to travel into the subsurface and back to the receiver) depends on what is referred to as the material's relative dielectric permittivity. This is the ability of a material to store an electrical charge. Different materials will conduct the GPR energy at different rates.

The intensity of the Reflected Energy or Amplitude of the Reflected Wave Parameter (AR) is dependent on the contrast between the relative dielectric constants or the contrast between two different materials as the radio wave travels from one medium to the next. The intensity of the reflected wave (Equation 1) shows this relationship at a boundary of two contrasting mediums (Clemena 2004).

$$
A R=A I \frac{\sqrt{\varepsilon_{r 1}}-\sqrt{\varepsilon_{r 2}}}{\sqrt{\varepsilon_{r 1}}+\sqrt{\varepsilon_{r 2}}}
$$

Where, AR is the intensity of the reflected energy

$\mathrm{AI}$ is the intensity of the incident wave

$\varepsilon_{\mathrm{r} 1}$ and $\varepsilon_{\mathrm{r} 2}$ is the relative dielectric constant of medium 1 and 2 

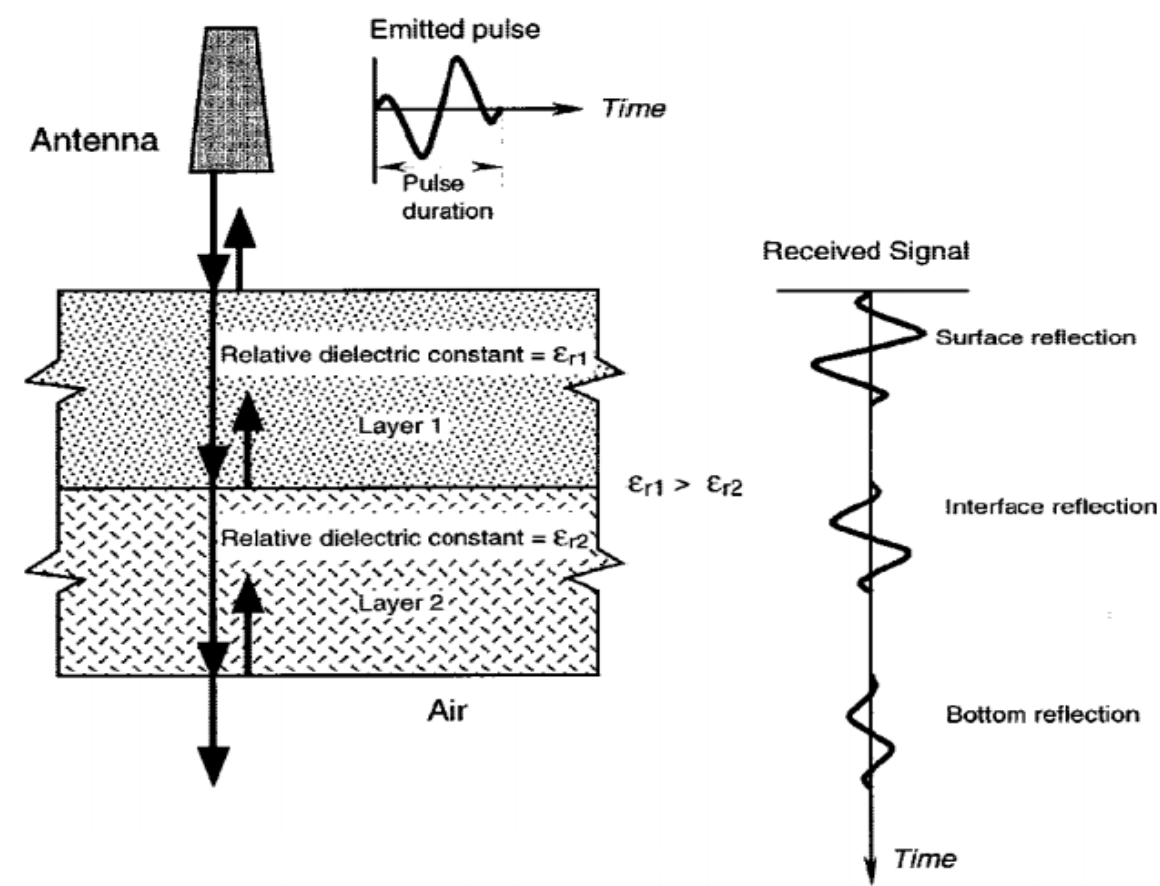

Figure 8: Principle of GPR reflection at the boundary

(Davis, 1998)

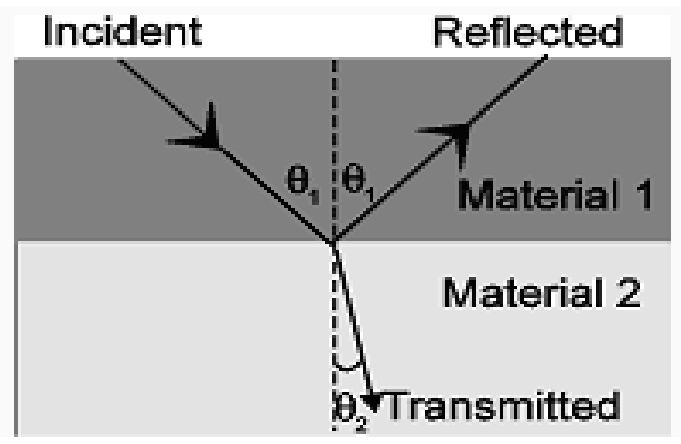

Figure 9: How GPR signals are transmitted and received at the boundaries.

(Sensors and Software, 2014) 
Table 2, illustrates the relative dielectric constants and velocities of various soil types as studied by (Davis, Anna and Daniels).

Table 2: Relative Dielectric Constants and Velocity of Various Soils.

\begin{tabular}{|c|c|c|c|c|}
\hline Material & $E_{r}$, Davis and Annan (1989) & $\varepsilon_{r}$, Daniels et al. (1995) & Velocity (m/ns) & Velocity (ftins) \\
\hline Air & 1 & 1 & 0.3 & 0.98 \\
\hline Distilled water & 80 & & 0.03 & 0.11 \\
\hline Fresh water & 80 & 81 & 0.03 & 0.11 \\
\hline Sea water & 80 & & 0.03 & $0.49-0.57$ \\
\hline Fresh water ice & $3-4$ & 4 & $0.15-0.17$ & $0.35-0.49$ \\
\hline Sea water ice & & $4-8$ & $0.11-0.15$ & $0.28-0.35$ \\
\hline Snow & & $8-12$ & $0.09-0.11$ & $0.35-0.50$ \\
\hline Permafrost & & $4-8$ & $0.11-0.16$ & $0.40-0.57$ \\
\hline Sand, dry & $3-5$ & $4-6$ & $0.12-0.17$ & $0.18-0.31$ \\
\hline Sand, wet & $20-30$ & $10-30$ & $0.05-0.09$ & $0.57-0.70$ \\
\hline Sandstone, dry & & $2-3$ & $0.17-0.21$ & $0.31-0.44$ \\
\hline Sandstone, wet & & $5-10$ & $0.09-0.13$ & $0.35-0.49$ \\
\hline Limestones & $4-8$ & & $0.11-0.15$ & 0.37 \\
\hline Limestone, dry & & 7 & 0.11 & 0.35 \\
\hline Limestone, wet & & 8 & 0.11 & $0.25-0.44$ \\
\hline Shales & $5-15$ & & $0.08-0.13$ & $0.33-0.40$ \\
\hline Shale, wet & & $6-9$ & $0.10-0.12$ & $0.18-0.44$ \\
\hline Silts & $3-30$ & & $0.05-0.13$ & $0.16-0.44$ \\
\hline Clays & $5-40$ & & $0.05-0.13$ & $0.16-0.44$ \\
\hline Clay, dry & & $2-6$ & $0.12-0.21$ & $0.40-0.70$ \\
\hline Clay, wat & & $15-40$ & $0.05-0.08$ & $0.16-0.25$ \\
\hline Soil, sandy dry & & $4-6$ & $0.12-0.15$ & $0.40-0.49$ \\
\hline Soil, sandy wet & & $15-30$ & $0.05-0.08$ & $0.18-0.25$ \\
\hline Soll, loamy dry & & $4-6$ & $0.05-0.08$ & $0.40-0.49$ \\
\hline Soil, loamy wet & & $15-30$ & $0.07-0.09$ & $0.22-0.31$ \\
\hline Soil clayey dry & & $4-6$ & $0.12-0.15$ & $0.40-0.49$ \\
\hline Soil, clayey wet & & $10-15$ & $0.08-0.09$ & $0.25-0.31$ \\
\hline Coal, dry & & 3.5 & 0.16 & 0.53 \\
\hline Coal, wet & & 8 & 0.11 & 0.35 \\
\hline Granites & $4-6$ & & $0.12-0.15$ & $0.40-0.49$ \\
\hline Granite, dry & & 5 & 0.13 & 0.44 \\
\hline Granite, wet & & 7 & 0.11 & 0.37 \\
\hline Salt, dry & $5-6$ & $4-7$ & $0.11-0.15$ & $0.37-0.49$ \\
\hline
\end{tabular}

(Baker, 2007)

This principle of relative dielectric constant can be used to determine if a strong reflection will be observed at the boundary or a weak reflection. Therefore, if an incident radar pulse travels from dry sand $\left(\varepsilon_{\mathrm{r} 1}=5\right)$ to wet sand $\left(\varepsilon_{\mathrm{r} 2}=30\right)$ this will produce a very strong reflection, when compared with the reflection from dry sand $\left(\varepsilon_{\mathrm{r} 1}=5\right)$ to limestone $\left(\varepsilon_{\mathrm{r} 1}=7\right)$, which has a value closer to zero (0). 
From (Equation 1). Assume constant (AI) for both case 1 and 2.

Case 1 dry sand $\left(\varepsilon_{\mathrm{r} 1}=5\right)$ to wet sand $\left(\varepsilon_{\mathrm{r} 2}=30\right),(\mathrm{AR})=-0.42$

Case 2 dry sand $\left(\varepsilon_{\mathrm{r} 1}=5\right)$ to limestone $\left(\varepsilon_{\mathrm{r} 2}=7\right),(\mathrm{AR})=-0.08$

Therefore, a faint reflection is expected at the boundary in Case 2. This can be pre-determined and used in post-data interpretation. It also means that most of the incident energy would refract and travel from medium 1 into medium 2 in Case 2.

\subsubsection{Electrical Conductivity (6)}

Another very important parameter of the medium being scanned (which affects the received GPR signal) is known as the electrical conductivity. This is a material property that determines the loss (absorption by soil) of (EM) energy as the wave passes through the mediums. Halabe found that (EM) waves of lower frequency can penetrate deeper into the medium with less resolution, while higher frequency waves penetrate to less depth, but provide better resolution (Halabe et al., 1993). This is an important factor to consider when selecting the antenna frequency for imaging both the target size and depth below the surface.

The depth of radar signal penetration is also affected by the water, mineral, and clay content of the soil. Lower material conductivity, such as found in sands and gravels, increase penetration of the radar signal, while fine grained, water saturated clays have high conductivity and will limit the penetration depth of the pulse. Salt water is very conductive and effectively blocks the radar signal from penetrating (Anna, 2003). Clay soils and saline water are a few limitations of GPR used in soil investigation, however, knowing of and understanding these limitations can be taken into consideration when interperting the data. 
The Conductivity of the medium can be determined by using equation 2 below:

$$
\alpha=1.69 \times 10^{3} \frac{\sigma}{\sqrt{\varepsilon_{r}}}
$$

Where, $\alpha$ is the attenuation of the signal $(\mathrm{dB} / \mathrm{m})$

$\sigma$ is the conductivity of the medium $\left(\Omega^{-1} \mathrm{~m}^{-1}\right)$

$\varepsilon_{\mathrm{r}}$ is the relative dielectric constant of the medium.

\subsubsection{Obtaining Depth from Time Window}

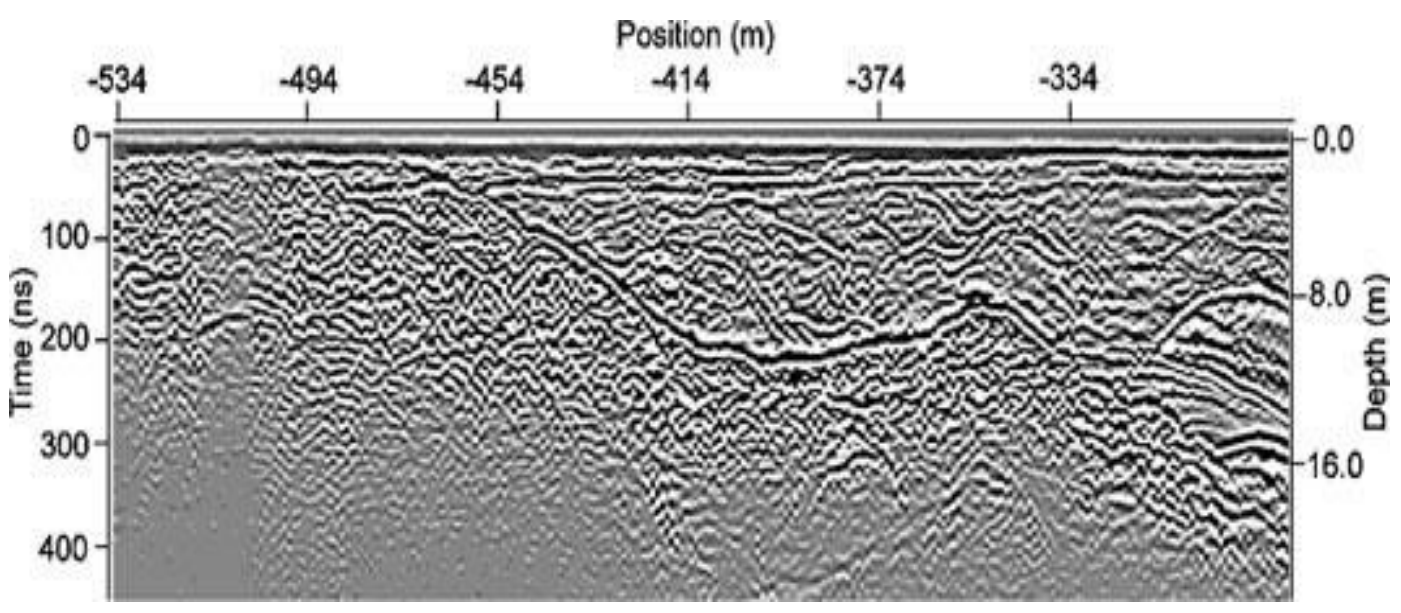

Figure 10: GPR data with time and depth on vertical axis

(Sensors and Software, 2014)

Figure 10 a cross-sectional plot of distance scanned along the horizontal x-axis (position) and its depth scanned along the vertical y-axis, is plotted and displayed on the monitor. These raw data images are also stored internally and can be transferred to computer systems for post-data processing and software manipulation.

The depth on the vertical axis is obtained from what is referred to as "travel time. This is the measured time for the bi-polar transmitted pulse to travel from the transmitter antenna into the ground, then reflected and detected by the receiver antenna. This travel time can be converted into depth by using Equation 3 once the velocity of the radar pulse is determined. 


$$
\text { Depth }=\frac{\text { Velocity } x \text { time }}{2}
$$

Eq.- 3

This in mind, the velocity at which the radar pulse travels through the material is dependent on the relative dielectric constants $\left(\varepsilon_{r}\right)$ of the material, which is different for various soil types and other non-metallic materials. The estimated velocity of the material can be obtained from Equation 4.

$$
\text { Velocity }=\frac{c}{\sqrt{\varepsilon_{r}}} \quad \text { Eq.- } 4
$$

Where, $\mathrm{c}=$ velocity of light $\left(3.0 \times 10^{8} \mathrm{~m} / \mathrm{s}\right)$

$\varepsilon_{\mathrm{r}}=$ relative dielectric constant of the material

From Table 2, the relative dielectric constant ranges from 1 (for air) to 80 (for water and other soil types). There are various values within this range, therefore, it can be said that the velocity obtained from equation 4 is an estimated velocity. This estimated velocity would transfer and error into the depth equation for Equation 3 in the order of $10 \%$ (Sensors and Software, 2014). Performing an on-site calibration would provide a more accurate estimate of the soil velocity; therefore, a more accurate depth axis could be obtained. This is done by performing a random walk-through with the GPR system, to locate targets that can be used along with a built in calibration tool to obtain the actual velocity of the soil type at the investigation site. 


\subsubsection{Collection GPR data}

Before conducting a GPR survey to collect soil data:

Step 1 is to conduct a desktop study to gain as much information about the site as possible. This can provide information on:

- the expected soil types to be encountered at the site,

- historical data, terrain and access of equipment,

- location and size of area to be scanned, and

- fiducials to aid in interpretation of collected data.

The desktop study, according to Sensors and Software should include reviewing the following if available:

- geological maps/and records,

- soil data and reports,

- utilities records,

- land use information and

- $\quad$ historical and archaeological data. (Sensors and Software, 2014).

Step 2 is to determine the depth of interest and target size eg. (cavities in limestone rock formation). This step is necessary to select the correct frequency antenna.

Step 3 is to estimate the velocity of the soil type as explained in section 2.4.3.

Step 4 is grid setup. Grids should be laid out with flags or pointers spaced equally across the site. Multiple grids may be used to collect larger data set. Before collecting grid data, test lines should be conducted to calibrate the GPR system.

Step 5 finally, is when the grid data can be collected. Detailed notes should be taken while collecting data. These notes can be used to aid in the interpretation of the GPR data. Grid and line data reflection mode, is normally used for GPR survey data collection, as illustrated in Figure 11, which uses constant offset reflection profiling with the Tx and Rx at a fixed separation distance as the unit moves across the surface of the site. 


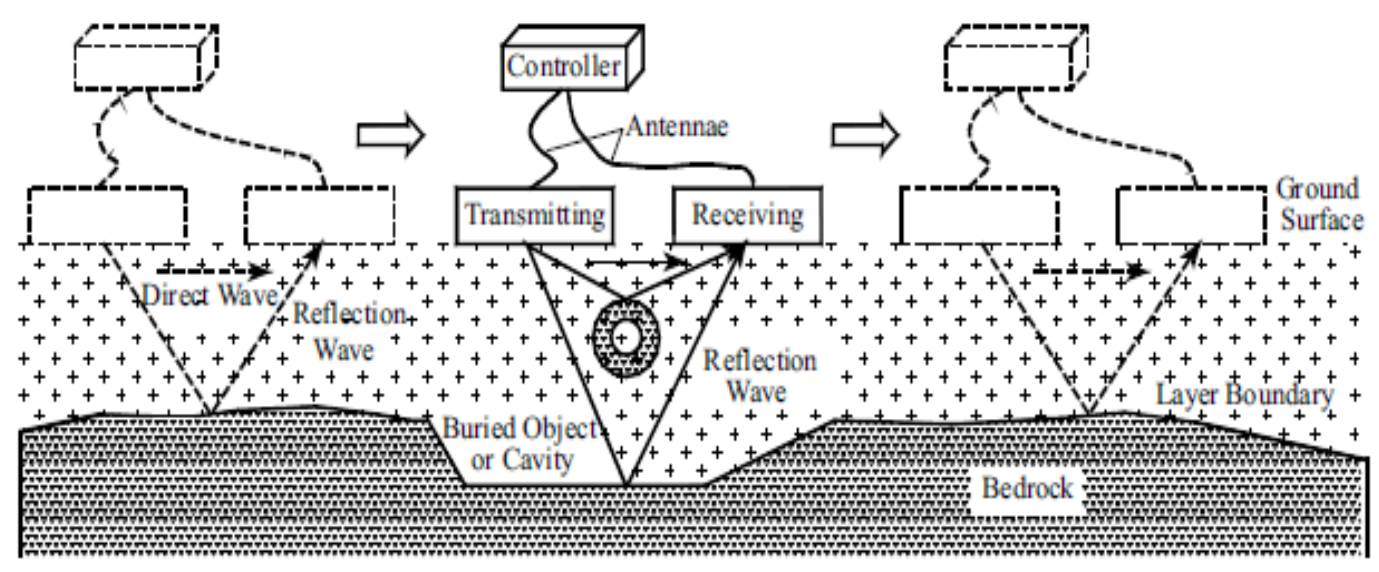

Figure 11: Constant antenna offset reflection profiling

(Yelf, 2006)

Another survey method used to collect GPR data is transmission mode or wide angle reflection and refraction (WARR) method studied in detail by (Annan 1985). This process involves fixing the position of the Tx and varying the separation of the Rx. As illustrated in Figure 12, WARR can be used for velocity calibration and improved resolution from the reflected radar pulses.

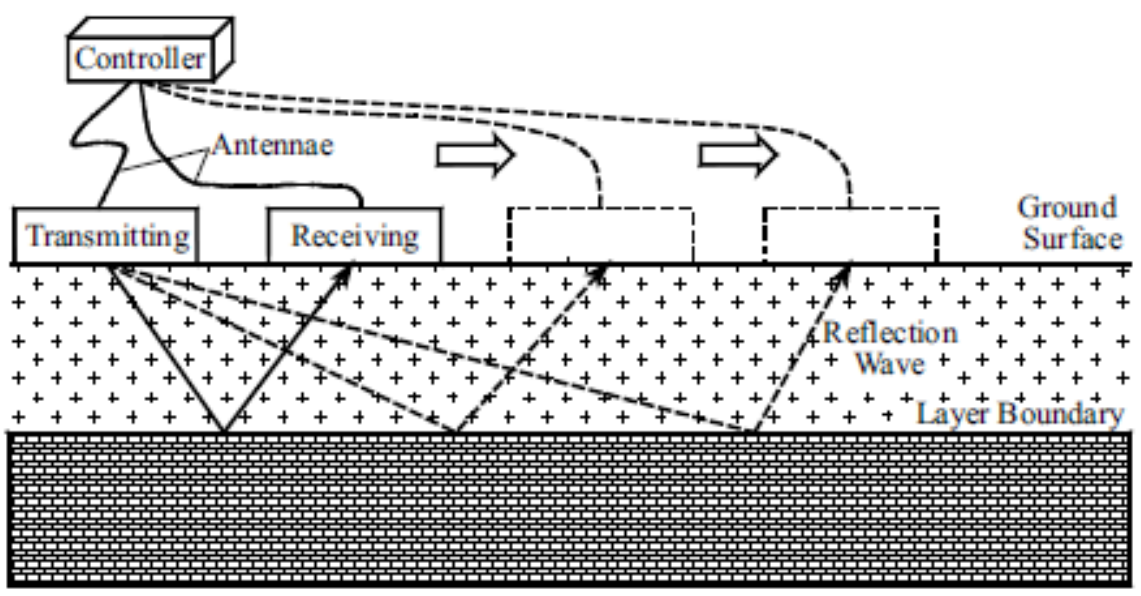

Figure 12: Transmission mode or Wide Angle Reflection and Refraction (WARR)

(Yelf, 2006) 
GPR can also be used in boreholes. These systems are called Borehole Radar (BH-GPR). These systems require special water-proof antennas to be inserted into the borehole. The boreholes can either be cased with Polyvinyl Chloride (PVC) casing or can be left uncased for cohesive soils. Metal casing prevents GPR signals from penetrating the soil from within the borehole.

BH-GPR surveys can be conducted in reflection mode, as illustrated in Figure 13a. This is done when a single borehole is available and both the Tx and Rx are placed within the same borehole. Reflections are recorded from contrasting di-electric material. When two or more boreholes are available, transmission mode ( see Figure $\mathbf{1 3 b}$ ) can be used to obtain a cross-hole measurement or a more detailed tomographic survey can be obtained by raising and lowering both $\mathrm{Tx}$ and $\mathrm{Rx}$ at various depths inside the borehole, as illustrated in Figure 13c.

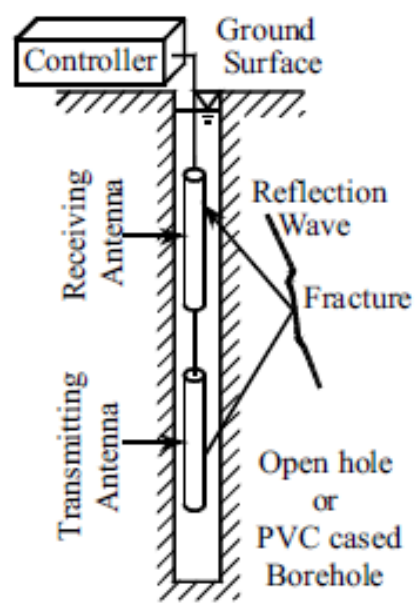

a) Single Hole Measurement

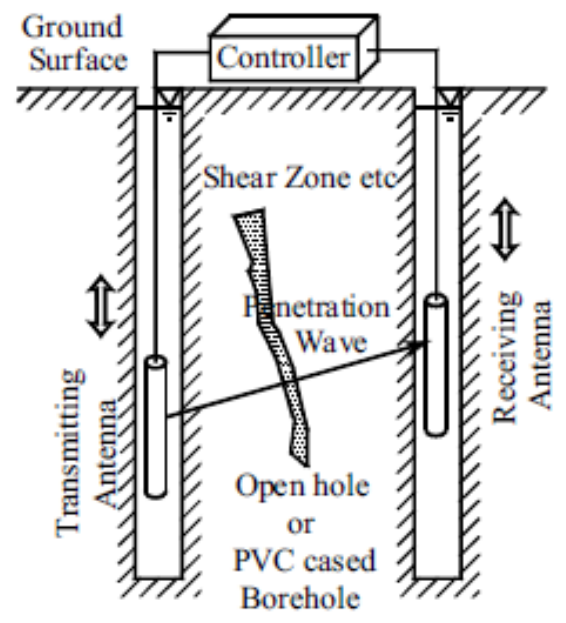

b) Cross Hole Measurement

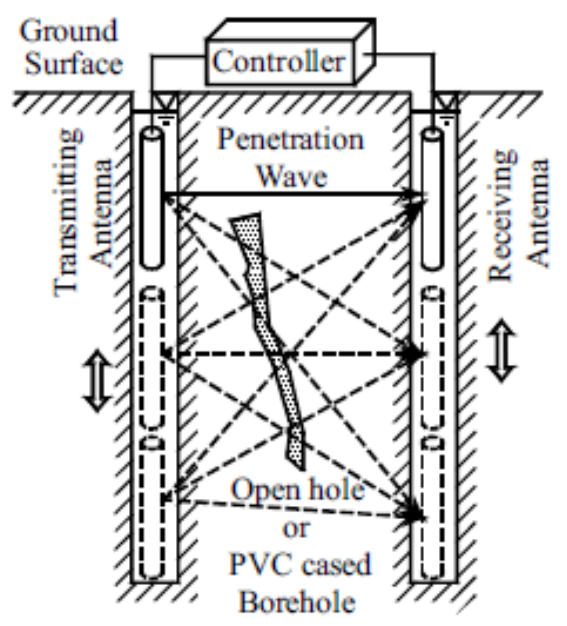

c) Radar Tomography

Figure 13: Methods of borehole GPR data collection

(Yelf, 2006) 


\section{CHAPTER 3 \\ APPLICATIONS IN GEOTECHNCAL ENGINEERING}

\subsection{Mining, Tunneling \& Quarrying}

GPR has found widespread use in the mining, quarrying and tunneling branches of engineering, it has the ability to detect changes in rock types and senses major changes in structures such as faults, fractures, and joints. Detailed applications include: outlining geological features, mineral exploration, determination of overburden thickness, evaluation of mine site, tunneling route selection designs, rock mass stability and defining ore zone.

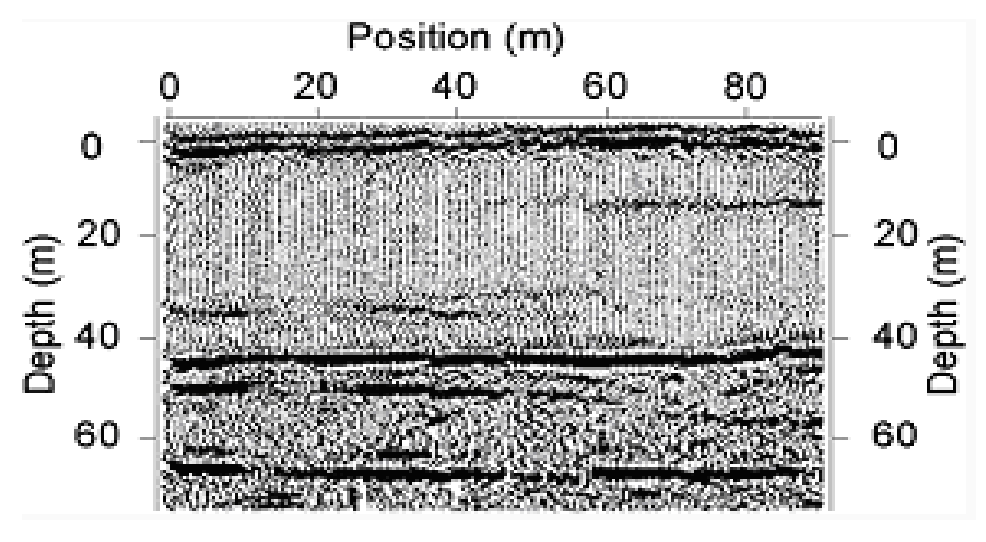

Figure 14: Data collected from a massive granite at 70m depth - black reflections are fractures.

(Sensors and Software, 2014)

\subsection{Ground Water Table, Karst Evaluation, and Soil Stratification}

GPR can define geologic strata and detect anomalous geological features below the surface. Applications in these areas ranges from route selection for roads, railways and pipelines to karst evaluations and contaminant plume mapping. It is also able to determinate depths- to bedrock, define-soil strata, and to identify karstic features and location of buried groundwater channels.

Sinkhole location surveys, which GPR can also be used for, are recommended around and under homes and buildings, over roadways, train tracks, airport runways, and in any other areas where the subsurface materials are subject to collapse. Sinkhole location surveys are conducted most 
often in karst limestone areas. However, these surveys may also be recommended in areas with soft soils or where mining or other subsurface disturbance activities have occurred.

Figure 15 below, shows the detection of a karstic cave formed in limestone. This cave was exposed during road constructions near Ben Shemen Israel. A GPR survey was done using the Pulse EKKO-IV system, manufactured by Sensors and Software located in Mississauga, Canada. It was later discovered, during archeological studies, that the cave was used to provide shelter during ancient Greek and Roman periods.

(a)

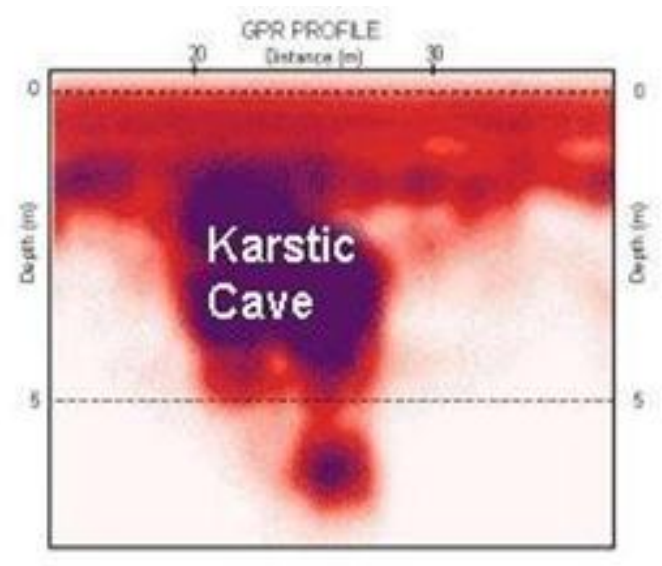

(b)

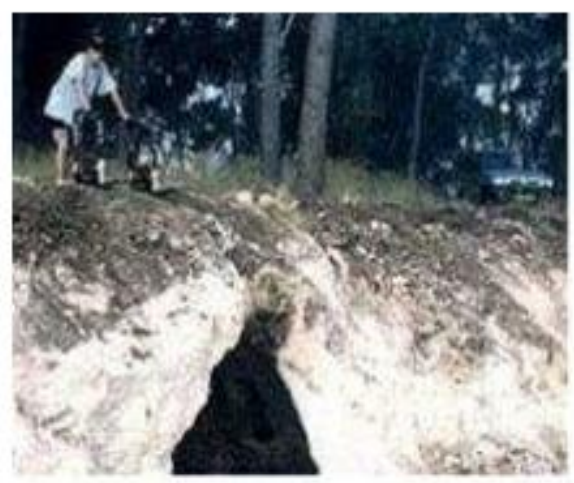

Figure 15: Detection of a karstic cave formed in limestone by post data processing (a), this cave was exposed during road construction (b)

Figure 16 and Figure 17 shows, how GPR was used to explain the stratigraphy of late Pleistocene gravel dunes in the Mountains of southern Siberia. Dune 1 (a) and Dune 2 (a) shows the GPR data collected with GPS corrections, allowing elevations of profile traces to be determined. Dune 1 (b) and Dune 2 (b) shows interpretations of the radar interfaces for Dune 1 and 2 respectively. Well-defined cross-sets of the radar stratigraphy shows bounding surfaces and the unconformable interfaces with the underlying gravel deposits. The palaeoflow right to 
left. The natural angle repose of the sand dunes could be extracted in both illustrations. (Carling, 2016)
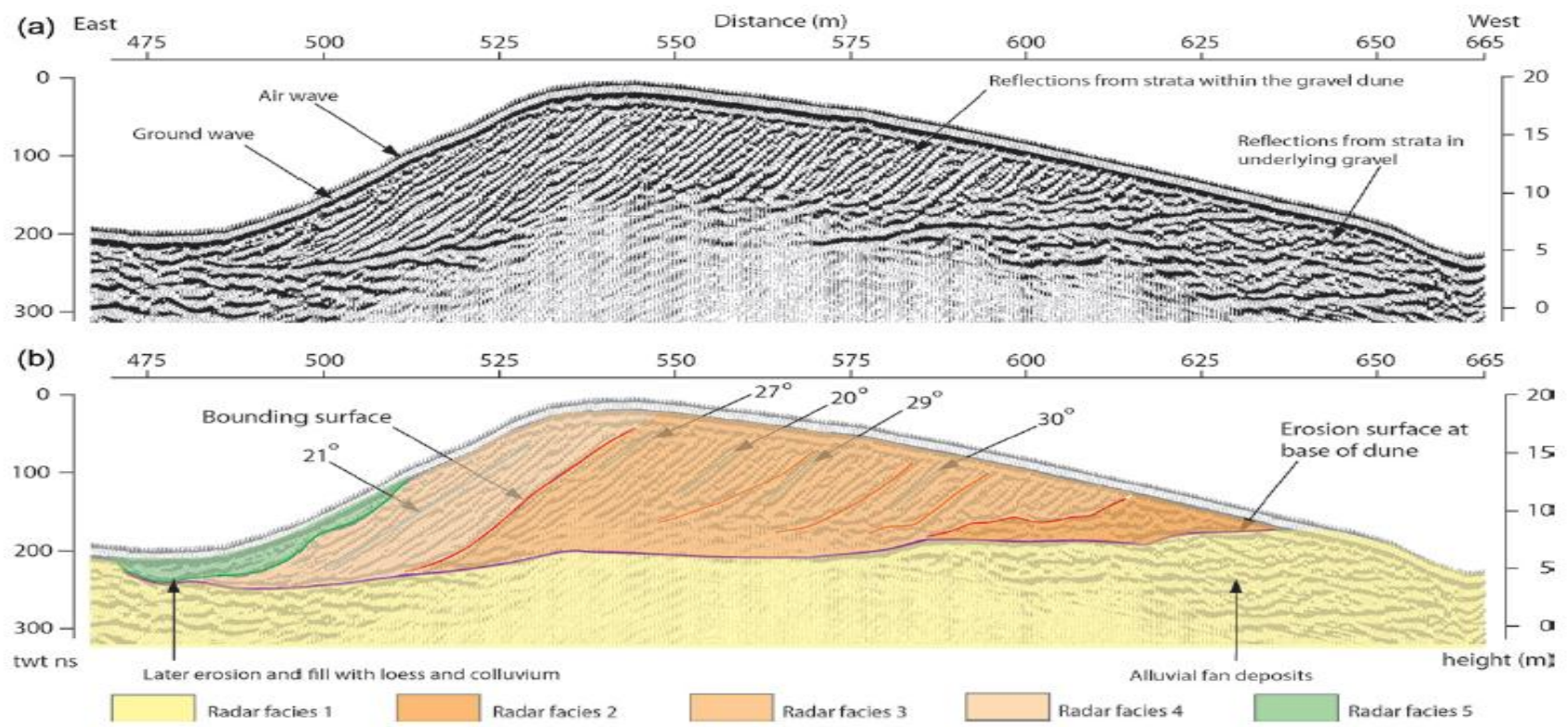

Figure 16: Dune 1, Ground Penetrating Radar Reflections (a), Interpretation of reflections (b)

(Carling, 2016)

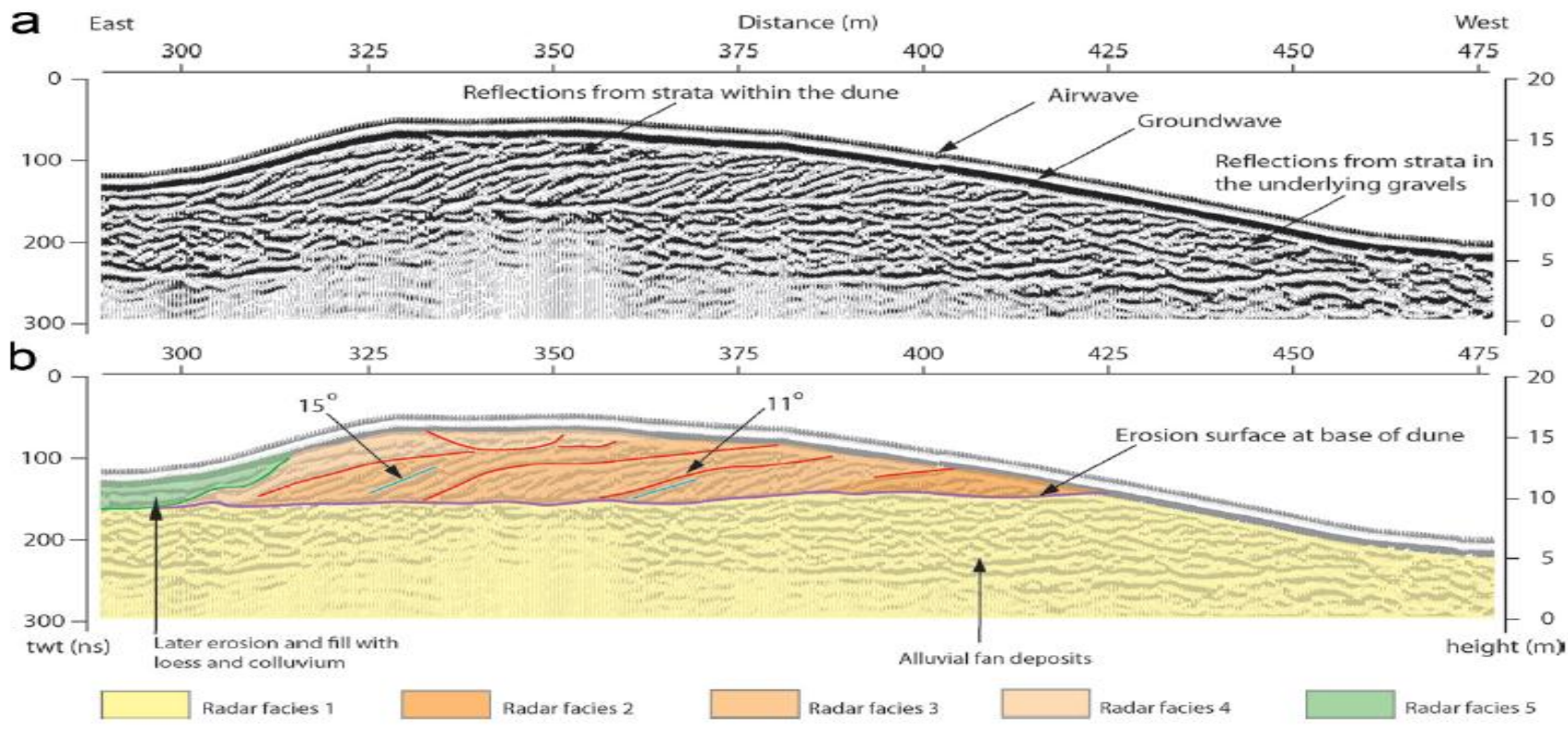

Figure 17: Dune 2, Ground Penetrating Radar reflections (a), Interpretation of reflections (b)

(Carling, 2016) 
Figure 18: Soil stratigraphic profile and water table interface.
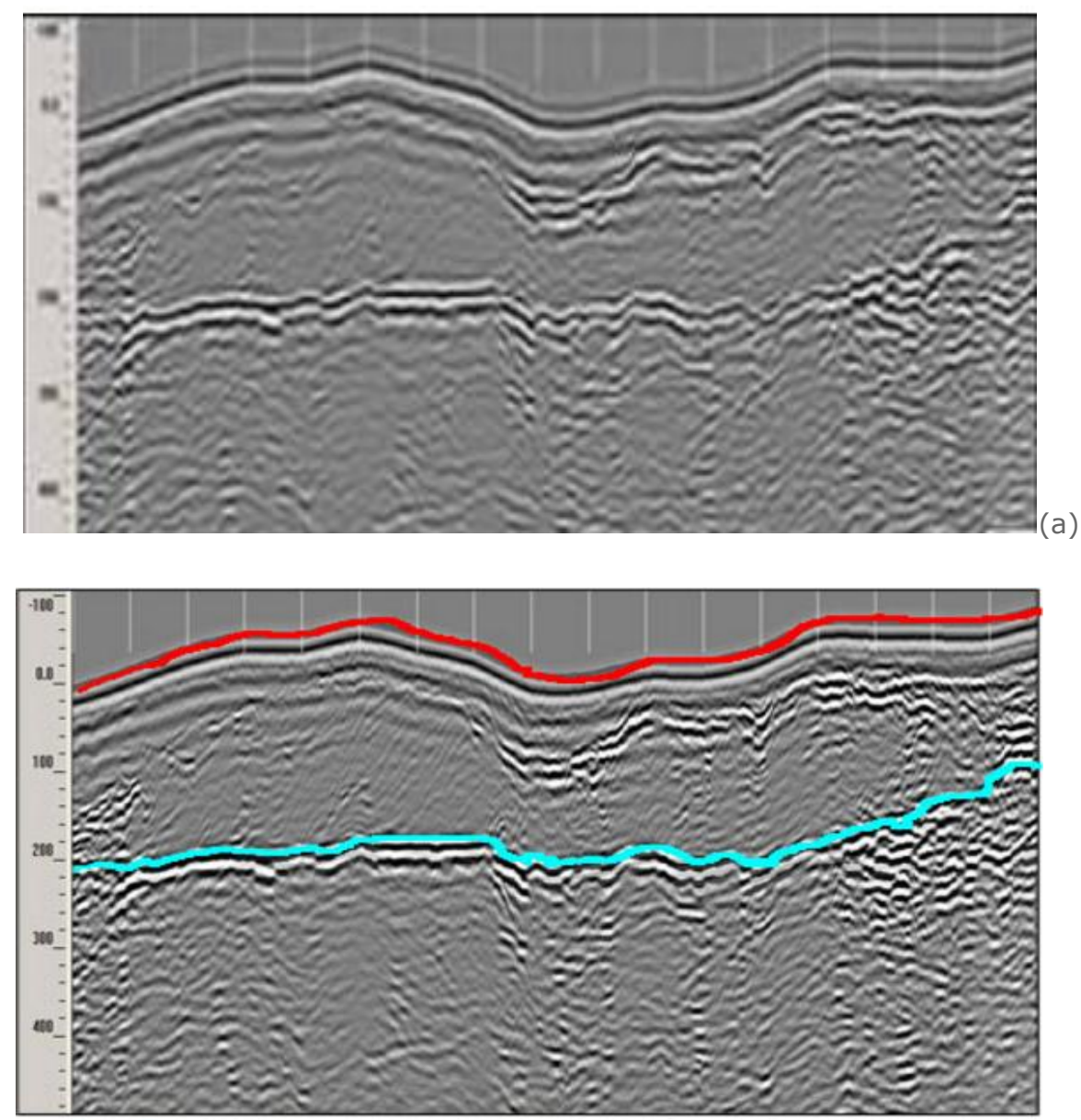

Corrected surface

Water table

(b)

Figure 18: Soil stratigraphic profile and water table interface

Profile data collected with GSSI, SIR 2 and $100 \mathrm{MHz}$ antenna indicating well-defined water table interface and stratigraphic cross-bedding.(GSSI, 2001) 
Figure 19 below illustrates, sub-bathymetry stratigraphic profile data collected by Sensors and Software Noggin $250 \mathrm{MHz}$ antennas. Many geotechnical investigations such as bridges or pipeline crossings need substantive sub-surface control data, reliable water depth and define sub-bottom structure. Depending on the salinity, radio waves can easily penetrate fresh water. The high contrast between the dielectric constant of water and other soils creates very good reflection at the boundaries (Sensors and Software, 2014). This data can be collected from a boat on the surface of the water.

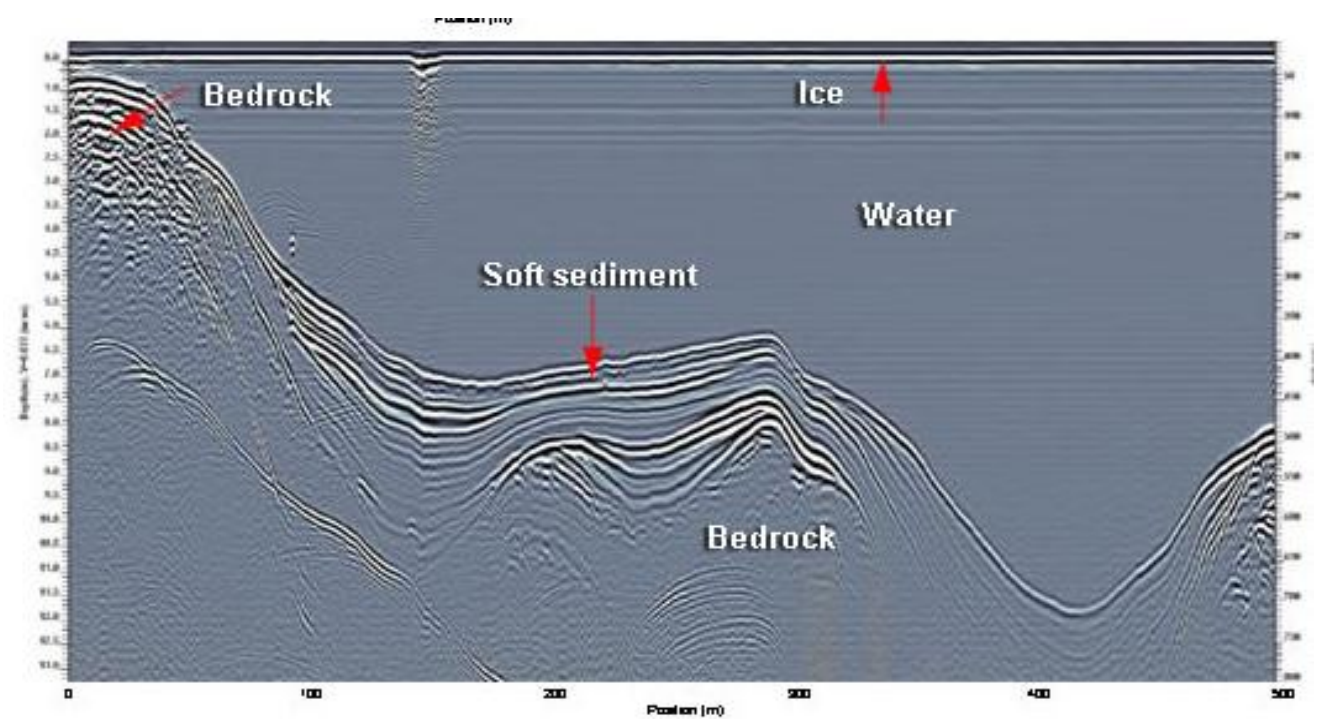

Figure 19: Sub-Bottom Stratigraphy Image in fresh water (Sensors and Software, 2014) 


\subsection{Quantitative Soil Parameters From GPR Data}

\subsubsection{Ground Penetrating Radar used to Detect Changes in Void Ratio Before and After Liquefaction Event.}

\subsubsection{Overview}

Robert E. Kayen et at. 1999, experimented with GPR at Treasure Island to identify changes in void ratio before and after a liquefaction event at a man-made geotechnical experimentation site in San Francisco Island. In this experiment control blasting was used to trigger a liquefaction event. Borehole radar method used on this site for GPR data collection, which produced a tomographic profile as previously described in 2.4.4. The changes in void ratio due to soil contraction during the liquefaction was modeled and then predicted settlement-based on radar estimates were compared with the observed settlement. (Robert E. Kayen, 1999)

\subsubsection{Problem}

Liquefaction susceptibility is highly influenced by the void ratio state and sedimentary texture of the soil. The void ratio of sandy soil is normally estimated indirectly through empirical correlations with both Standard-Penetration-Test (SPT) and Cone-Penetration-Test (CPT) results. Depending on budget availability for the project, the void ratio can be estimated directly from laboratory analysis of frozen samples or gamma-ray density. Logging these procedures is expensive. Experimenting with the use of GPR provides a low-cost alternative that also provides quantifiable data for void ratio. 


\subsubsection{Results}

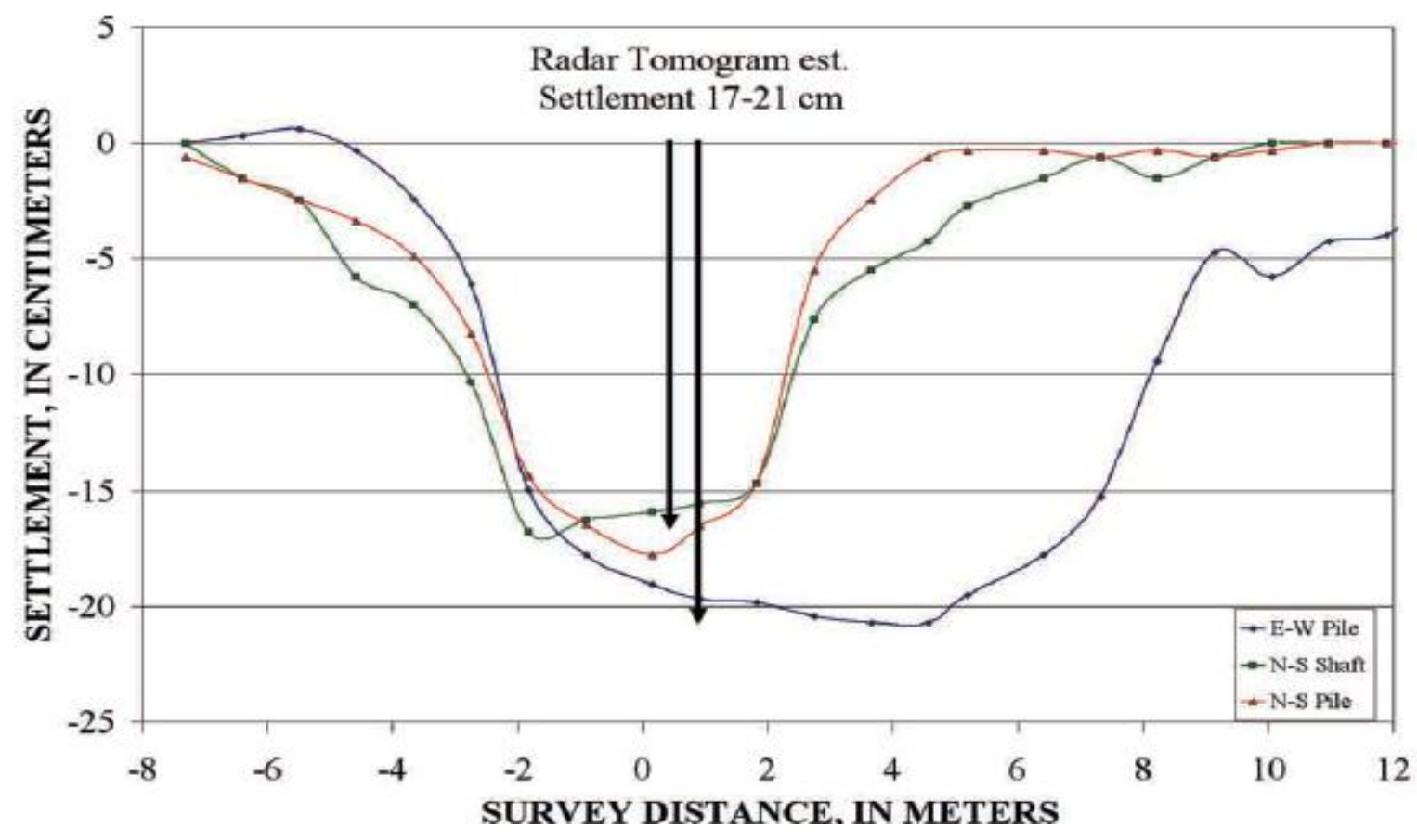

Figure 20: Results showing GPR predicted settlement vs. observed settlement.

(Robert E. Kayen et al., 1999)

\subsubsection{Conclusion}

The cross-hole GPR tomographic profile was able to produce quantifiable spatial details for the initial soil void ratio and the void-ratio changes due to a liquefaction event.

The radar-based estimates of volumetric strain associated with the void ratio changes resulted in settlements ranging from 17 to $21.3 \mathrm{~cm}$. These estimates are remarkably close to the groundlevel changes observed by survey methods before and after blasting 16.7 to $20.7 \mathrm{~cm}$, which is an independent measurement of the volumetric strain. 


\subsubsection{Ground Penetrating Radar used to Determine Soil Water Content.}

\subsubsection{Overview}

J. A. Huisman et at. 2002, reviewed the four methods used to extract soil water content from GPR data. The methods reviewed were, soil water content determined from reflected wave velocity, soil water content determined from ground wave velocity, soil water content determined from transmitted wave velocity and soil water content determined from the surface reflection coefficient. The principles, data quality and limitation of each method were reviewed, the results from soil water content obtained from transmitted wave volicity in borehole GPR is illustrated in Figure 21 which was applicable to this project. The other methods reviewed were related to ground surface moisture content relating to watershed runoff areas and agricultural studies.

\subsubsection{Results}

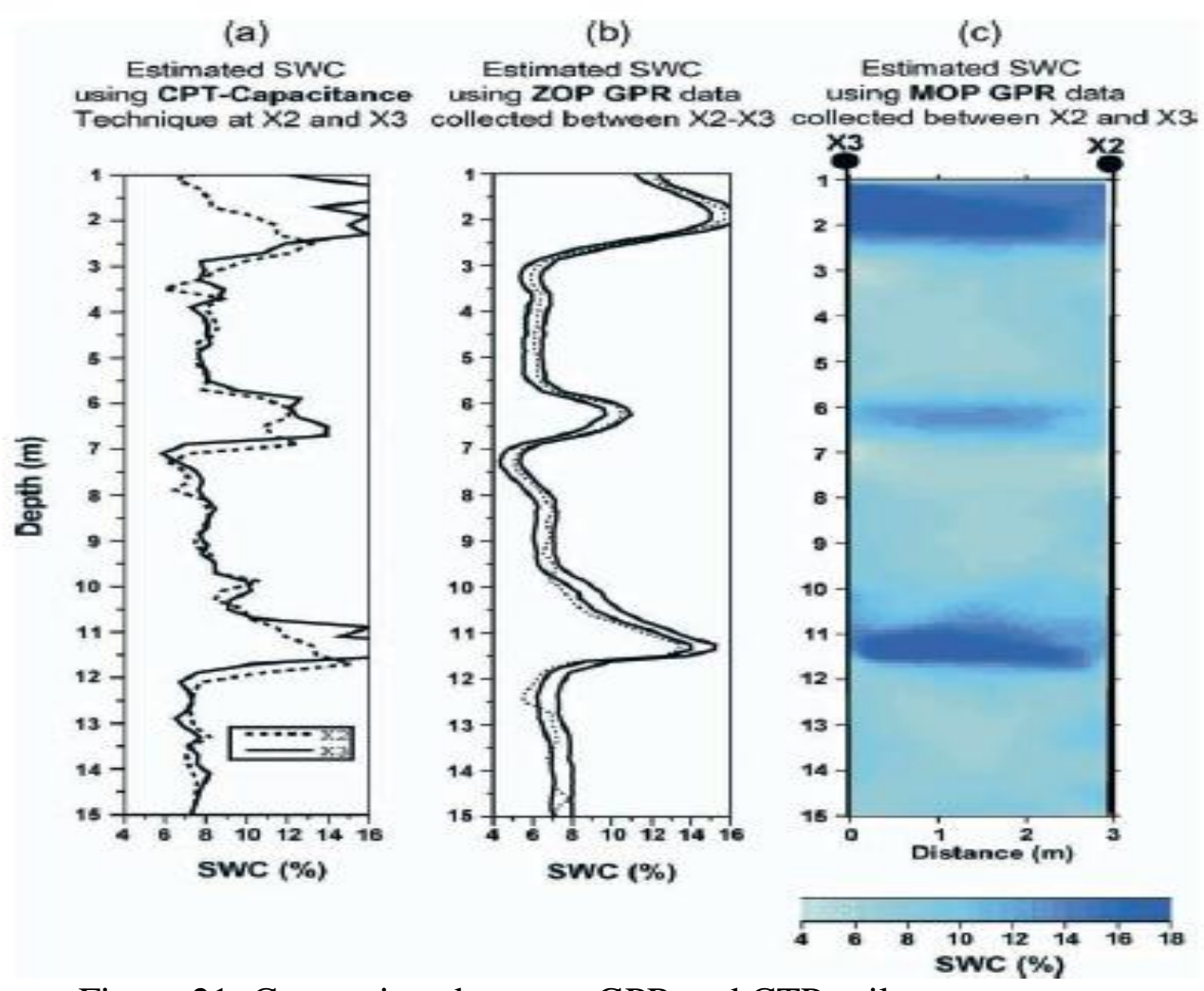

Figure 21: Comparison between GPR and CTP soil water content.

(Majer et al., 2002). 


\subsubsection{Conclusion}

From Figure 21, the results compares Cone Penetrometer Test (CPT) results with the estimated results of soil water content (SWC) obtained from a $200-\mathrm{MHz}$ antenna using zero offset profiling (ZOP) and multi-offset profiling (MOP) survey method of BH-(GPR).

Both GPR borehole survey methods provided similar soil water content (\%), at similar depths, when compared with the results of traditional CPT test. These results can conclude that BH-GPR is a viable tool to accurately measure soil water content. 


\section{CHAPTER 4}

\section{OBSERVATIONS, ANALYSIS AND REPORT FOR HOTEL EXPANSION, JAMAICA}

\subsection{Overview}

The following report was deduced from field observations, data processing, and interpretations made from GPR data collected at a hotel expansion project located in St. Ann, Jamaica. The site was located along the north west boundary of Jamaica which is predominantly limestone rock formation.

\subsection{Problem}

A geotechnical firm in Jamaica where I reside was consulted to provide geotechnical report for a Hotel Expansion Project located in the North West region of the island. The SPT and coring bore-log data determined the soil types across the site to be calcareous, gravelly sand, and limestone rock formation over medium to hard coral calcareous sandy gravel/rock. Based on observations of small visible voids from the surface during the site clearance and geotechnical borings, it was confirmed the existence of cavities and potential karst formation may exist across the site and the immediate areas adjacent to the site. The geotechnical engineers sought-to know the scope and spread of cavities across the proposed buildings along with their location, size and depth.

A geophysical company was contracted to determine the answers to the above problem by using Ground Penetrating Radar.

A special thank you to Mr. Courtney Simons (Field Technician) and WCI team who allowed me to observe and take notes during their investigation. 


\subsection{Background Information Prior to GPR Surveys}

The GPR team expected to find limestone, clays, water, and air during their data collection, which was based on observations and review of drilling logs. These soil types have very different dielectric properties and would result in a high contrast reflection at the interfaces. Clays generally exhibit electrical properties of velocity " $\mathrm{v}$ " $(\mathrm{m} / \mathrm{ns}) 0.08$, conductivity “ $\sigma$ " $(\mathrm{mS} / \mathrm{m}) 2$ 1000, attenuation “ $\alpha$ " (dB/m) 1-300 and dielectric permittivity " $k$ " 5-40 @ 100MHz, Meanwhile limestones generally exhibit electrical properties of velocity " $\mathrm{v}$ " (m/ns) 0.12 , conductivity “ $\sigma$ " (mS/m) 0.5-2, attenuation " $\alpha$ " (dB/m) 0.4-1 and dielectric permittivity " $k$ " 4-8 @ 100MHz while air exhibit electrical properties of velocity " $\mathrm{v}$ " (m/ns) 0.30 , conductivity “ $\sigma$ " $(\mathrm{mS} / \mathrm{m}) 0$, attenuation " $\alpha$ " $(\mathrm{dB} / \mathrm{m}) 0$ and dielectric permittivity " $\mathrm{k}$ " $1 @ 100 \mathrm{MHz}$. The distinct clay/silts, air, water and limestone properties at contacts should provide excellent contrast for (GPR) survey profile lines.

The expected intensity reflections at soil interface boundaries are calculated and shown in Table 3, these values were determined from (equation 1) and (table 2) detailed in section 2.4.1.

Table 3: Calculated expected contrast intensity at boundaries.

\begin{tabular}{|c|c|c|c|c|c|}
\hline Soil Medium & Er1 & Er2 & Al & AR & Comment \\
\hline Dry limestone to air & 7 & 1 & 1 & 0.45 & Very good Reflection very visible \\
\hline Dry limestone to dry sand and gravel (filled cavity) & 7 & 5 & 1 & 0.08 & Poor Reflection, additional processing required \\
\hline Dry limestone to dry clay/silts (filled cavity) & 7 & 4 & 1 & 0.14 & Fair Reflection \\
\hline Wet limestone to wet sand and gravel (filled cavity) & 8 & 25 & 1 & -0.28 & Fair Reflection, visible \\
\hline Wet limestone to water (filled cavity) & 8 & 80 & 1 & -0.52 & Very good Reflection very visible \\
\hline Wet limestone to wet clay/sitts (filled cavity) & 8 & 30 & 1 & -0.32 & Good Reflection, very visible \\
\hline
\end{tabular}

The above AR value from Table 3 represents the reflection intensities at the various soil boundary interfaces expected for the site. These values were calculated using equation 1, table 2 and excel spreadsheet, to input values of the relative dielectric permittivities of the various soil types that were expected to be encountered. 
The engineers were concerned about depth of influence (up to 10+ $\mathrm{m}$ and a minimum cavity size of $1 \mathrm{~m}$ ). Once the background information was gathered, the GPR team selected suitable grids based on access/ scope areas, and line spacings. Antenna frequency was also selected to conduct the survey.

\subsection{Methodology}

A total of four (4) grids were established to carry out GPR data acquisition grid 0 ( $24 \times 49 \mathrm{~m})$, grid $1(16 \times 39 \mathrm{~m})$, grid 4 (7 x 28m) and grid 5 (13 x 32m), shown in schematic illustration Figure 24. The data was collected using a Noggin Smart Cart GPR system, by Sensors and Software Inc., Mississauga, Canada. The method used for data collection was constant antenna offset reflection profiling as described in section 2.4.4. The antenna had center frequency of 100 $\mathrm{MHz}$ for deep soil profiling. A series of single line test were completed to optimize acquisition parameters, and these results were used to design a 2-D survey. The step size data was $1.0 \mathrm{~cm}$, this is the distance travelled between each transmitted pulse and the transmitter-receiver separation data is $0.5 \mathrm{~m}$, this is the fixed distance between the transmitter and receiver. 


\subsection{Data Collection}

The image below, shows the area selected for the new building A construction, lines in yellow represents the scope area for the GPR investigation (including Grids 0, 1, 4 and 5).

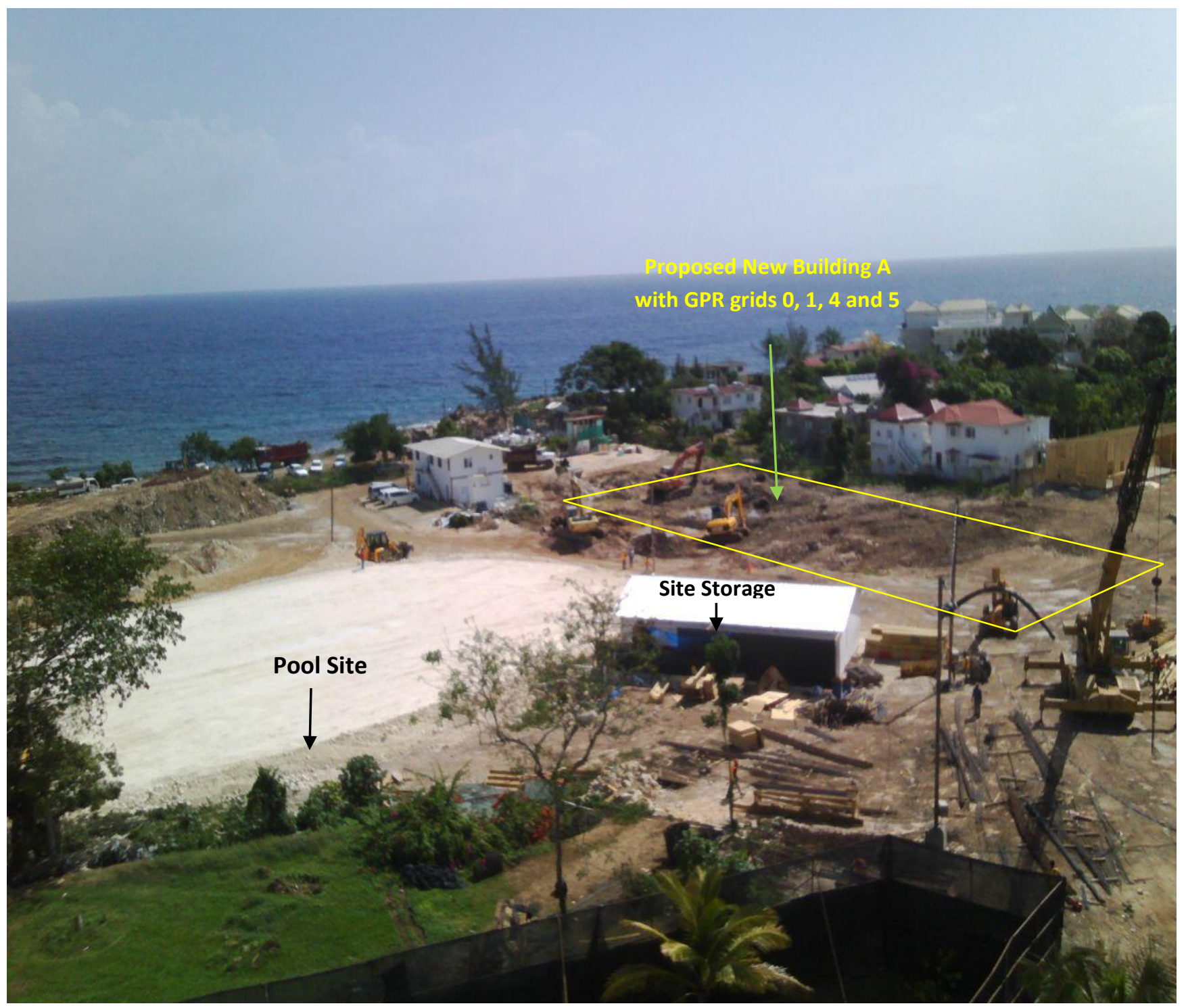

Figure 22: Showing site layout, existing structures and equipment onsite during data collection. 
During the site walk through a number of observable surface features were noted. The spread, orientation and depth of these cavity features are unknown. The GPR survey seeks to provide these answers.

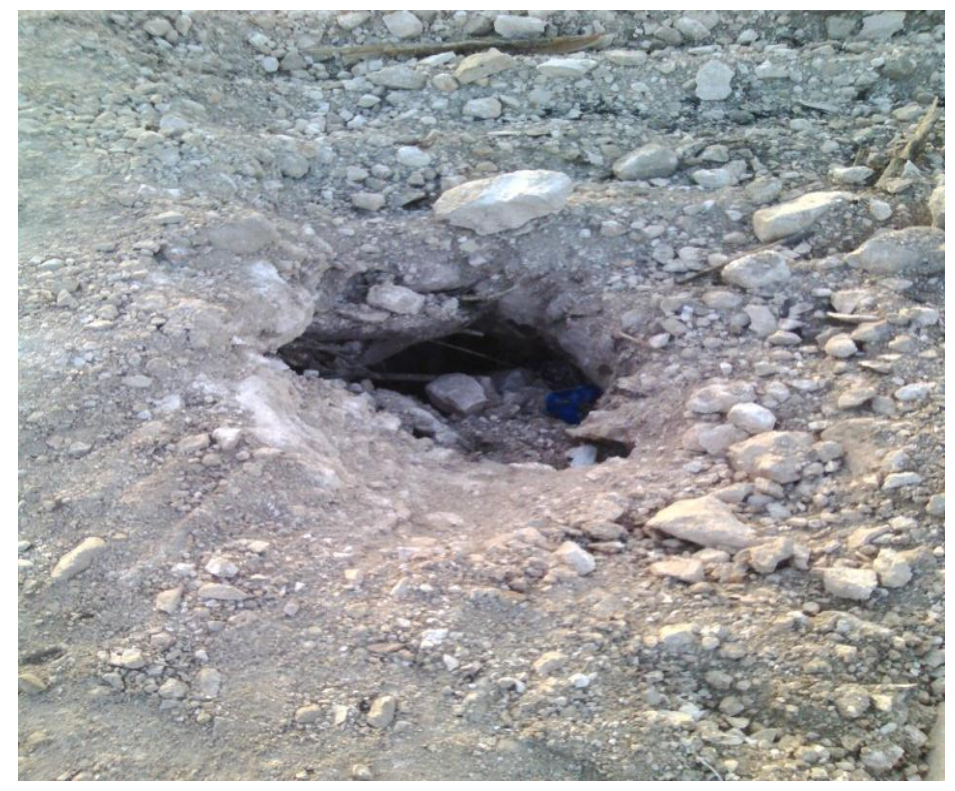

Picture 1: Observable void from surface 
The image below shows the Noggin Smart Cart GPR system used during data collection. The wheeled system allows for rapid data collection in open areas. The particular system used was fixed with Noggin $100 \mathrm{MHz}$ antennas for deep soil profiling. The yellow monitor or the Digital Video Logger (DVL) provides real-time images of the subsurface; it is equipped with highresolution touchscreen that provides flexible data collection settings. The system is approximately $20 \mathrm{lbs}$ in combined weight.

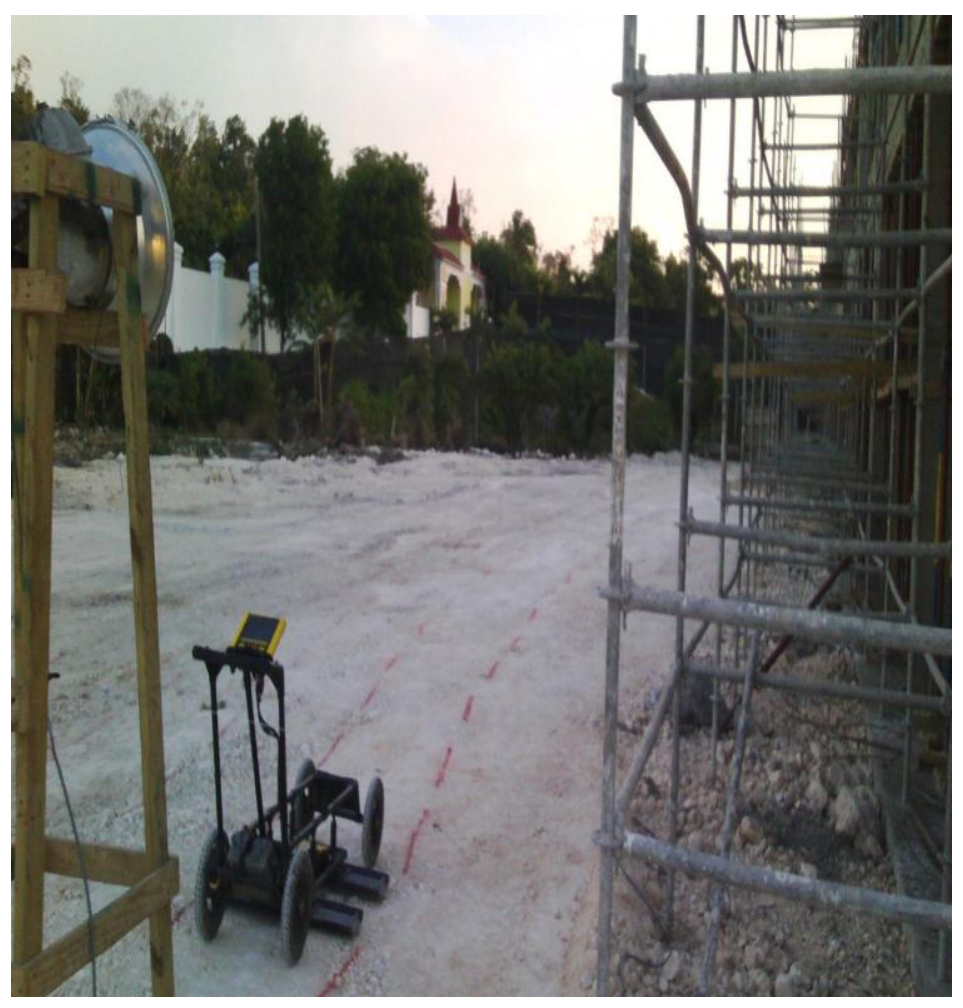

Figure 23: Noggin Smart Cart GPR machine 100MHz antenna during data collection 


\subsection{Results, Data Processing and Analysis}

The data collected was processed by Sensors and Software's Ekko_Project Software Version 3 with the slice view module option enabled. Depth slice view module allowed the user to view the reflections in plan at depth intervals below the surface. Complex targets and interpretations can be differentiated by using the slice view module. (Sensors and Software, 2014)

The main processing steps to better understand and visualise the GPR data were narrowed down to five.

\section{Time zero correction:}

This allows the user to set the first reflection event usually from the ground surface to zero (0) doing this allows depth to targets and interfaces to be more accurate.

\section{Back ground filters:}

This option removes any horizontal banding collected in the data normally due to interference.

\section{Band passes filters:}

This option allow high and low band frequency signal to be separated from the central frequency providing more clarity to the data.

\section{Gain:}

This option allows the user to increase or decrease the amplitude of the collected data, hence better visualization of target features.

\section{Measuring wave velocity:}

This option allows the user to calibrate the velocity of the soil by using hyperbola matching from identified target. Correct velocity is related to accurate depth to target estimation.

(Bigman 2017) 
Figure 24 is a schematic illustration of the grid positions and locations with respect to the site. Each grid has a referenced starting point $(\mathrm{y}=0$ and $\mathrm{x}=0)$. The image also shows the direction in which the data was collected for each grid.

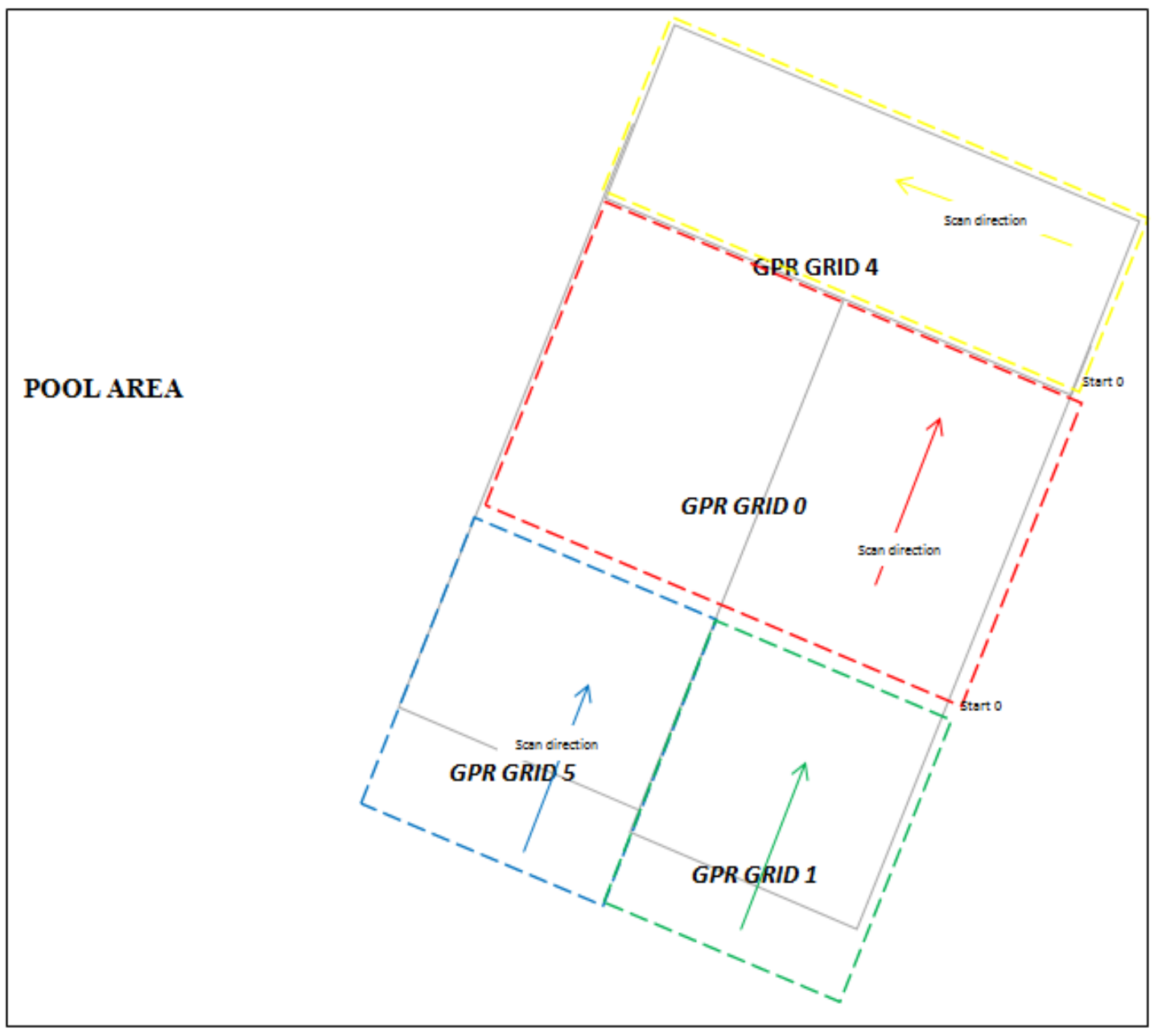

Figure 24: Schematic Illustration of Building A GPR Grid Layout 


\subsection{1 $\quad$ Results Grid 0}

Figure 25 shows, the GPR profile lines collected in grid 0 . A total of 23 lines were collected each ending at approximately $48 \mathrm{~m}$ in trace length. Each line was started at the same reference line along the $\mathrm{x}$-axis. This will ensure consistency in identifying sub-surface interpretation and features across the site.

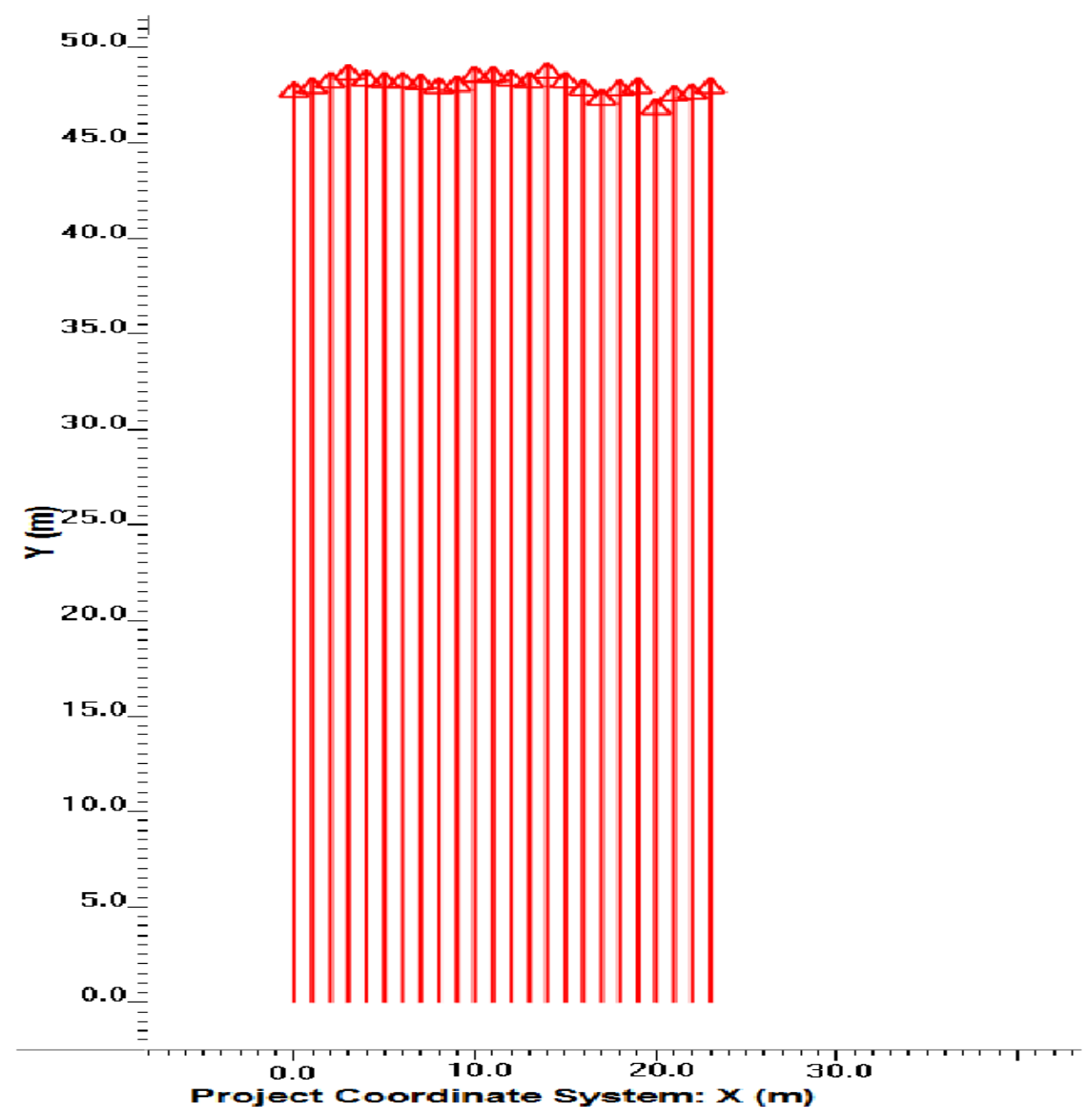

Figure 25: Illustrating profile lines collected in grid 0. 
Figure 26, below was obtained from the slice view module within the Ekko_Project version 3 software. The slice view module allows the user to slice up and down in depth through the 3D data cube. Both plan and sections can be view simultaneously, making difficult targets more recognizable.

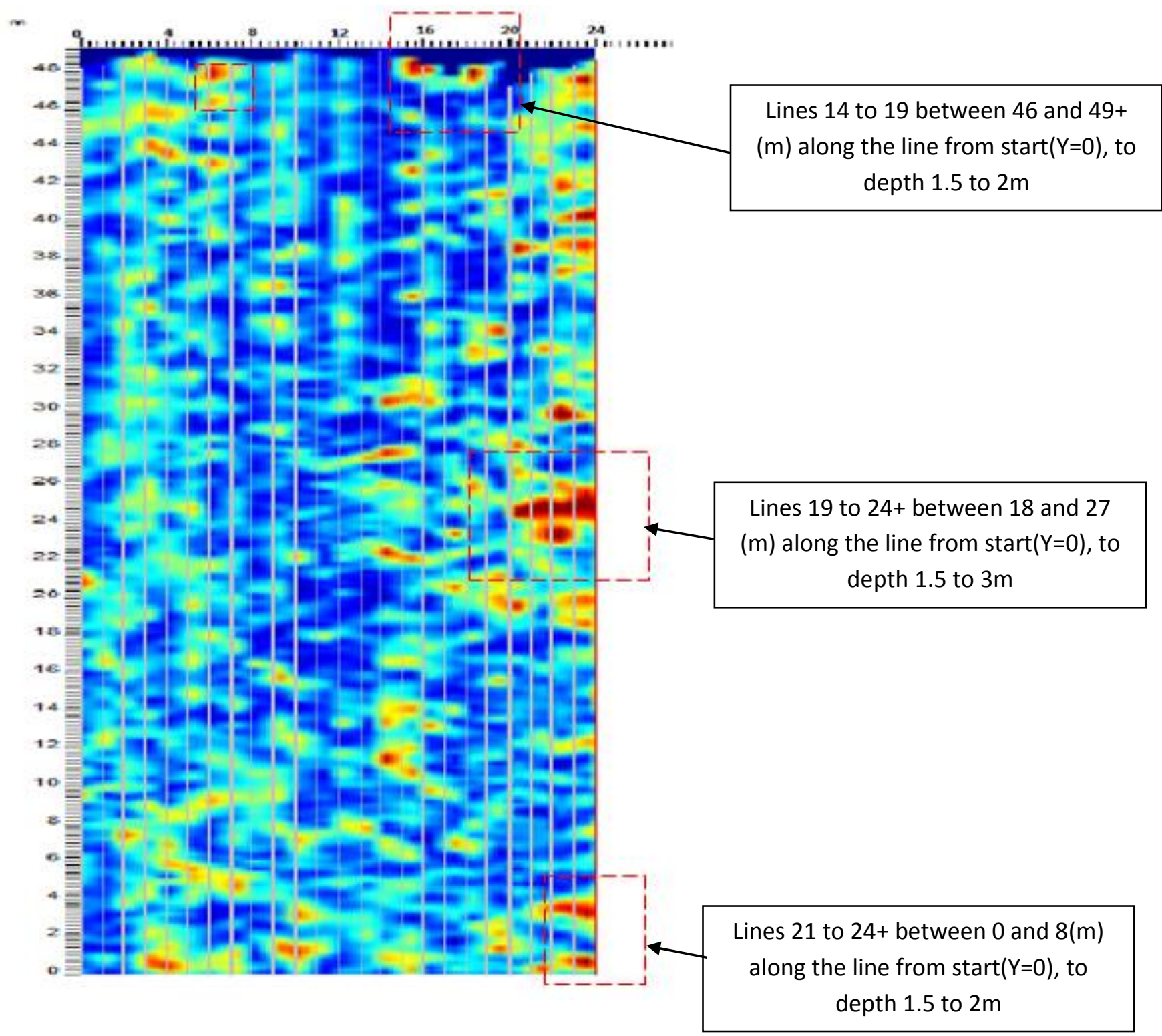

Figure 26: Horizontal Slice across Grid 0 at 0.50- $0.75 \mathrm{~m}$

In Figure 26 the main areas suspected of voids are highlighted in red dotted lines. All areas of concern exceed grid boundaries as represented the in figure. 
Figures 27, 28 and 29, illustrates the cross sections through the GPR profile lines collected at lines 17,23 and 24 respectively.

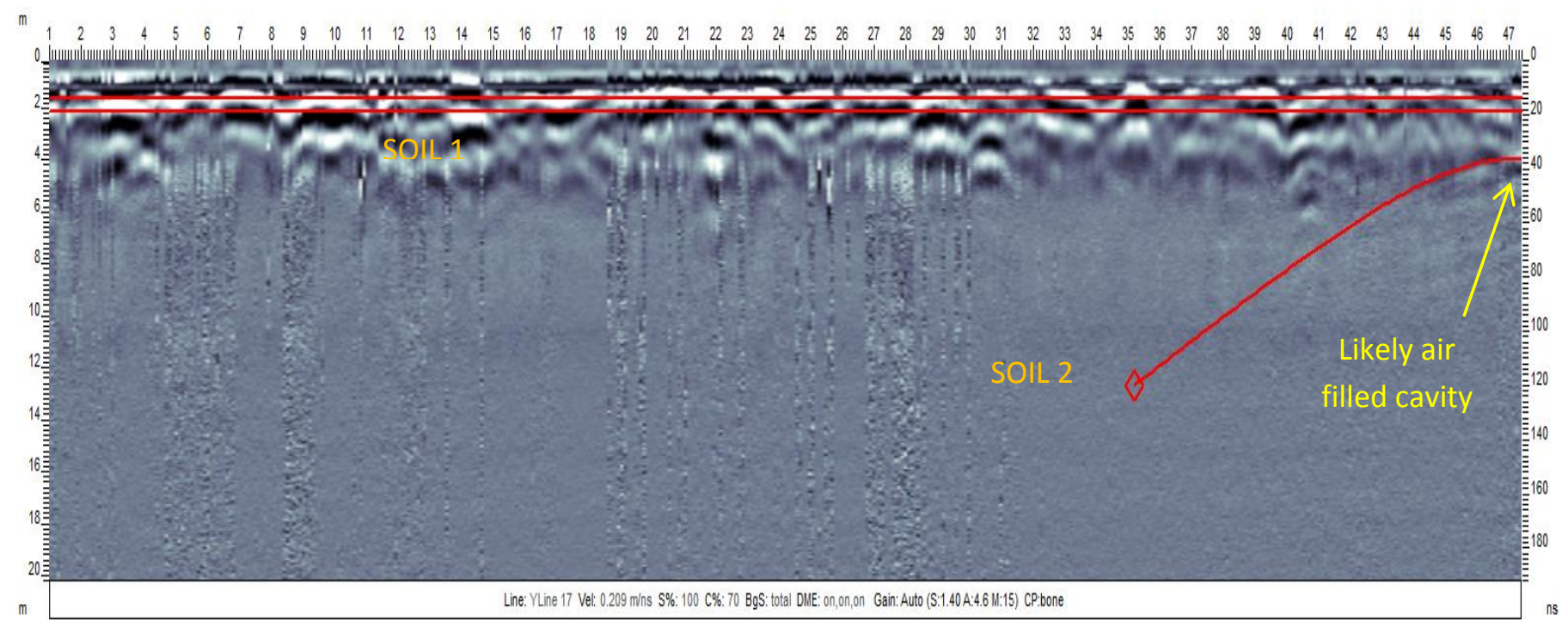

Figure 27: Processed vertical section of line 17 profile line indicating slice lines at $1.50-2.00 \mathrm{~m}$

In Figure 27, there are likely targets between 46 and 49+ (m) along the line from start 1m, at approximate depth of 2 to $5 \mathrm{~m}$ also seen in line 14 to 19 , based on the GPR data, the GPR machine did not fully reach over the target during onsite data collection, however partial reflection from the target ahead of the machine was received and the calibrated velocity of $0.209 \mathrm{~m} / \mathrm{s}$ was at target indicating the presence of air at target.

Two main soil types can be identified in Figure 27. Soil 1 and Soil 2, GPR cannot determine the composition of the soils types, however it can be seen that Soil 1 exist up to an approximate depth of 5m. Reflections from Soil 1 are highly variable and can be assumed to be highly fractured limestone rock with pockets of air. Correlating GPR data with borehole log data will provide more accurate interpretations of subsurface features. 


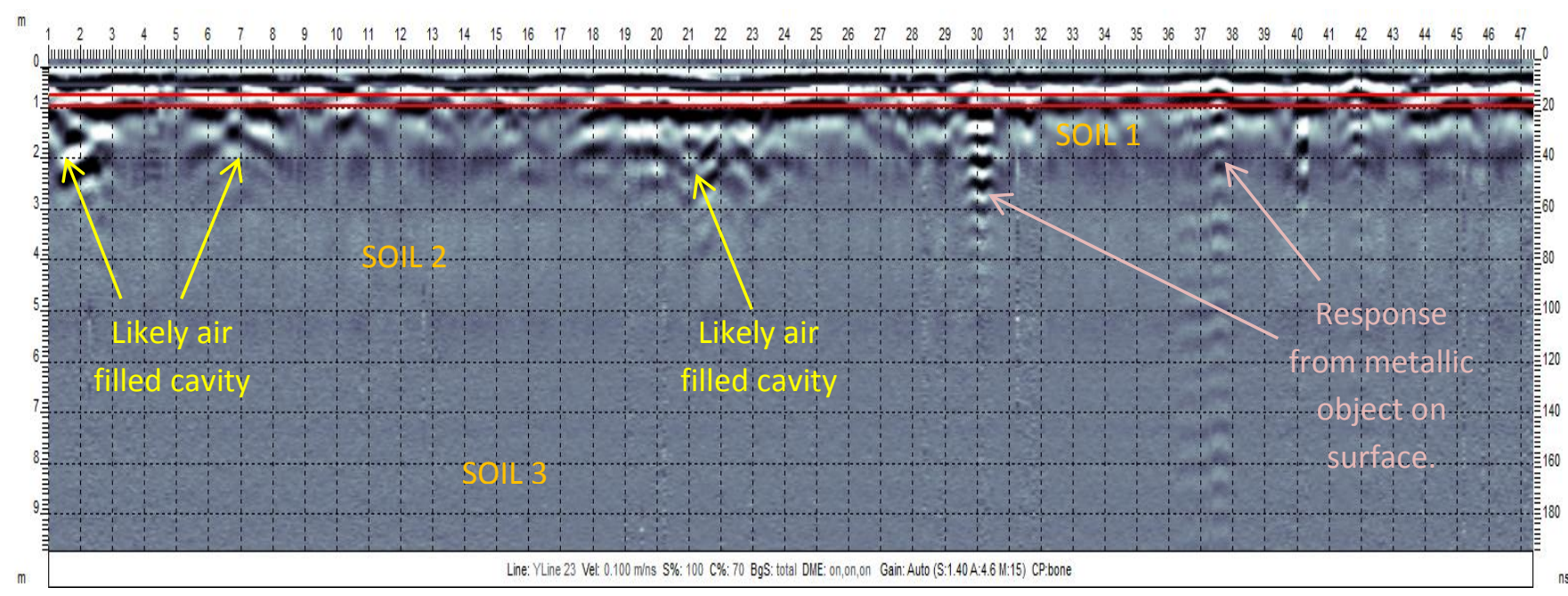

Figure 28: Vertical section of line 23 profile line indicating slice lines at $0.75-1.00 \mathrm{~m}$

In Figure 28, likely target exist between 0 to $8 \mathrm{~m}$ and 18 to $24 \mathrm{~m}$ along the line from start 0 , at depth 1-3m. These features are also seen in lines 21 to24+ and lines 19 to 24+ respectively. Strong multiple responses to metallic objects are noted at 30, 37.5 and $42 \mathrm{~m}$ along the line.

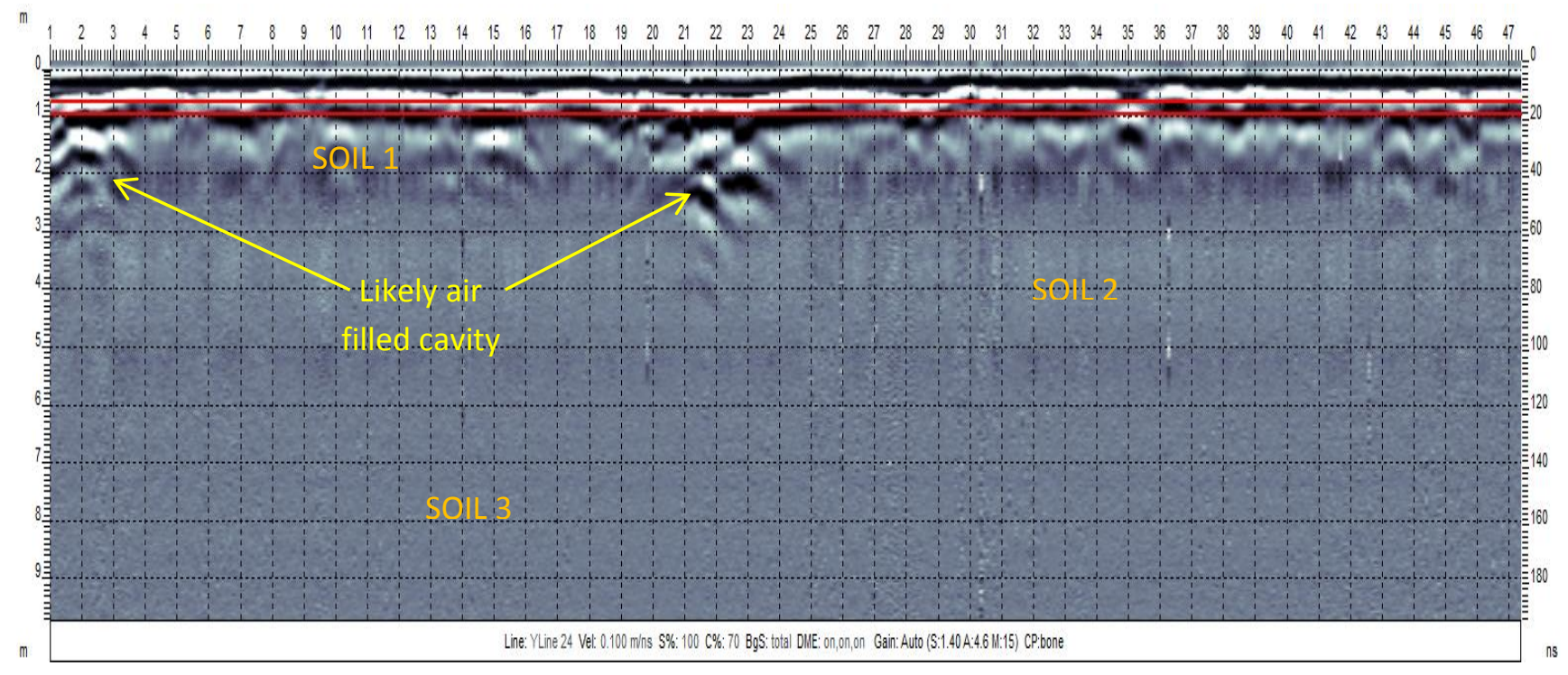

Figure 29: Vertical section of line 24 profile line indicating slice lines at $0.75-1.0 \mathrm{~m}$

In Figure 29, likely target between 0 to $5(\mathrm{~m})$ and 19 to $24 \mathrm{~m}$ along the line from start $1 \mathrm{~m}$, at depth $1-3 \mathrm{~m}$ and 1 to $4 \mathrm{~m}$ respectively. These targets were noted also in lines 21 to $24+$ and 19 to $24+$ respectively indicating a lateral spread of the target features. 
The above procedures and methods interpretation were repeated for grids 1, 4 and 5 .

\subsubsection{Results Grid 1}

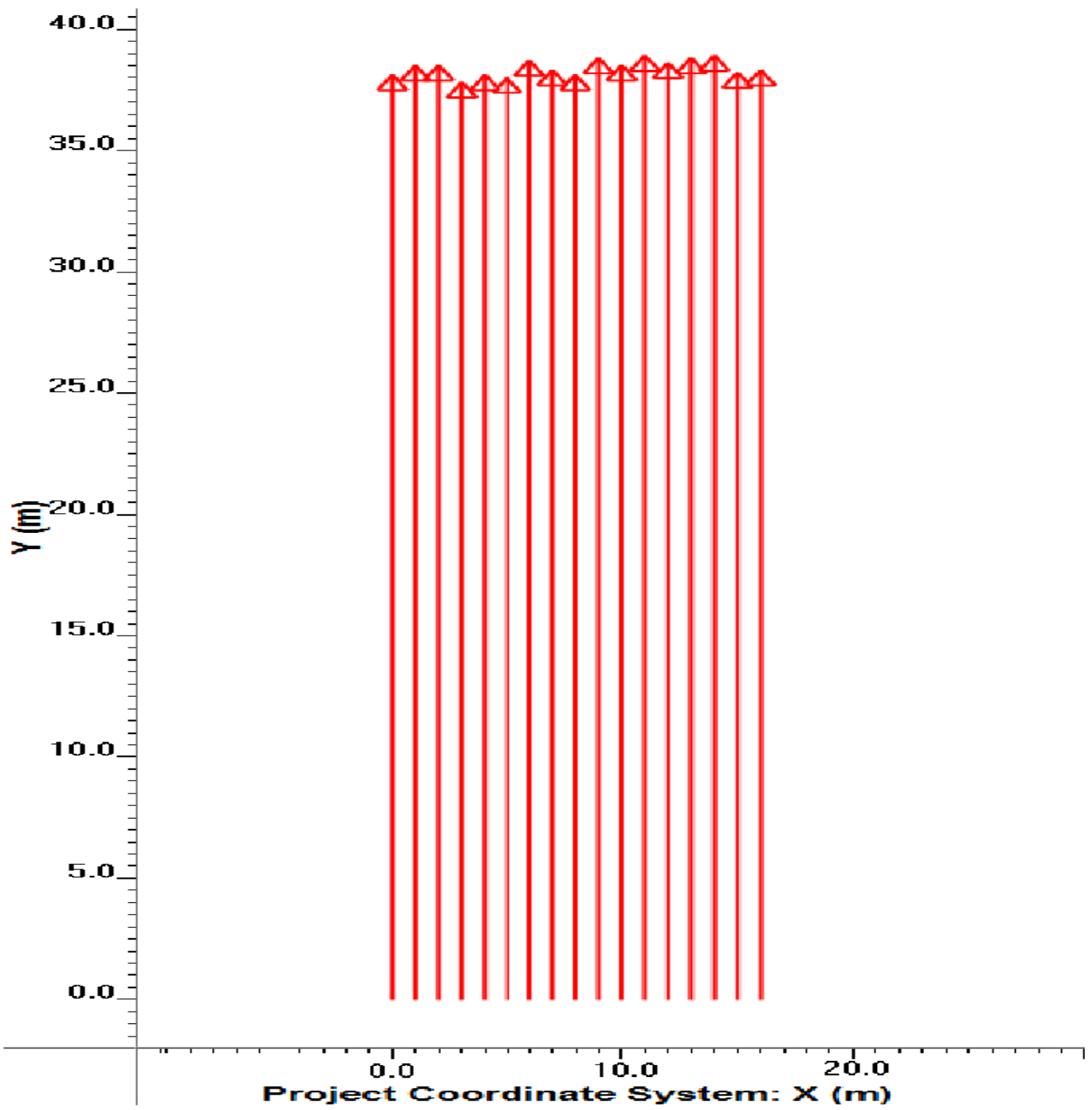

Figure 30: Illustrating profile lines collected in grid 1 


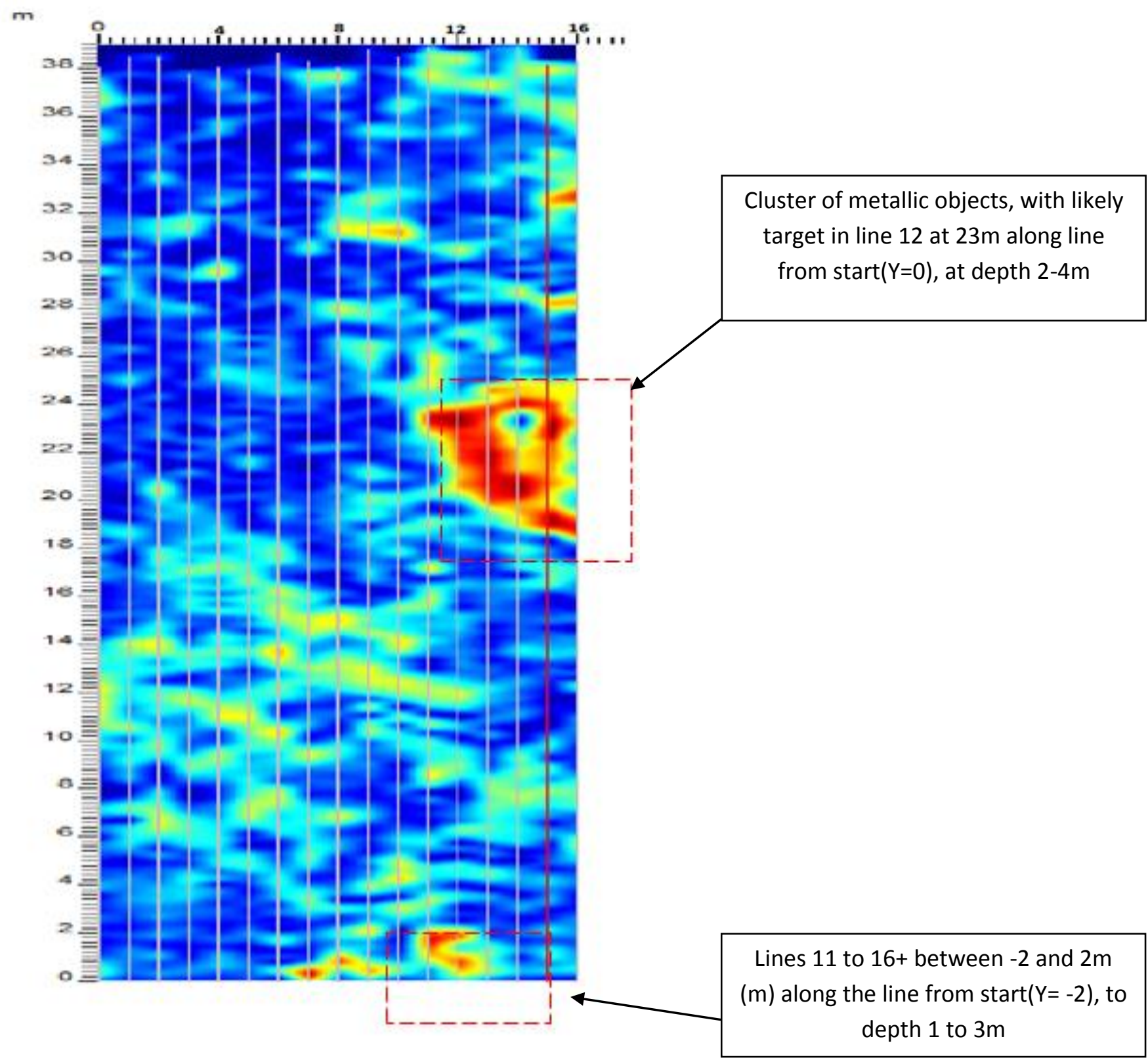

Figure 31: Horizontal Slice across Grid 1 at $0.50-0.75 \mathrm{~m}$ depth

Figure 31 illustrates, the main area of concern across the grid, which are highlighted in red dotted lines. All areas of concern exceeded the grid boundaries as represented in figure. 


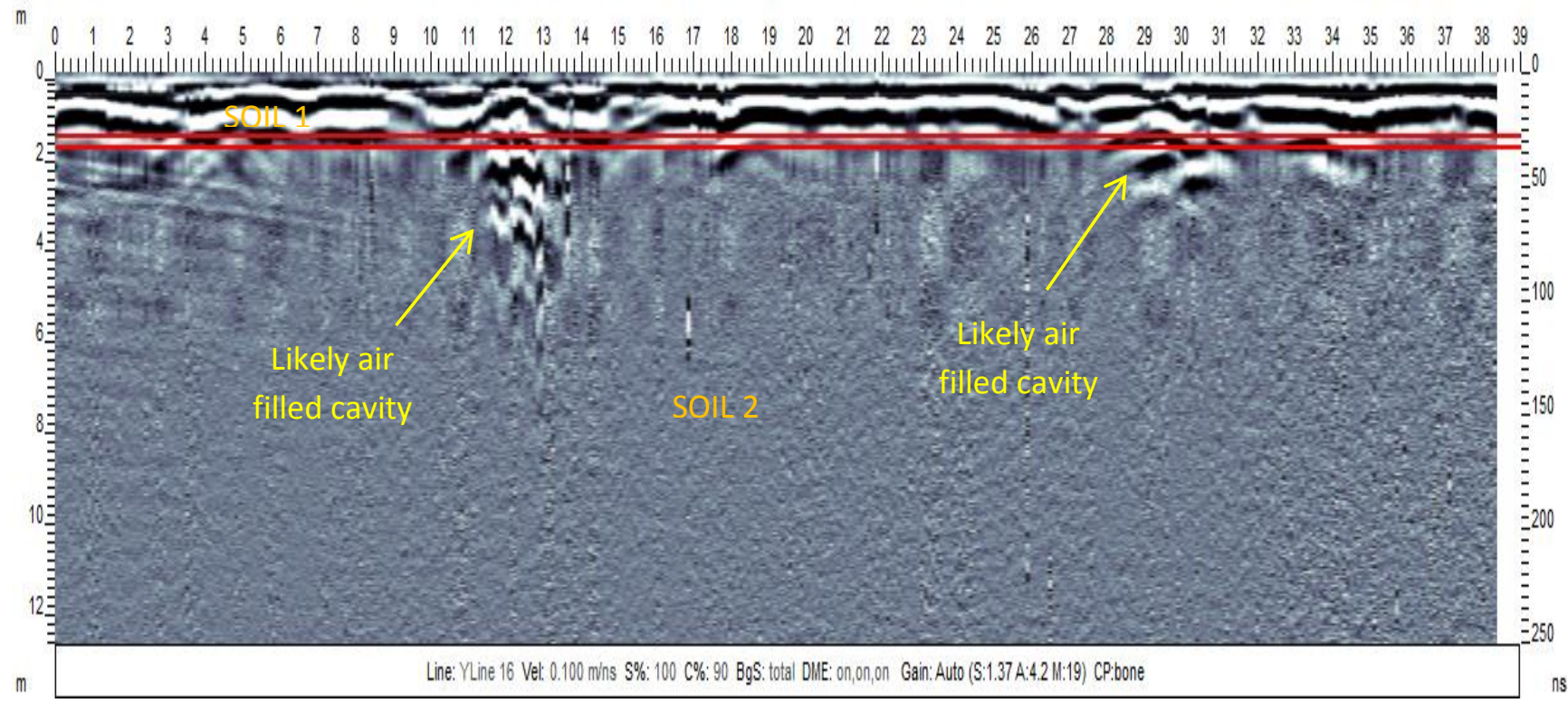

Figure 32: Vertical section of profile line 16 with slice lines at 1.50 to $1.75 \mathrm{~m}$

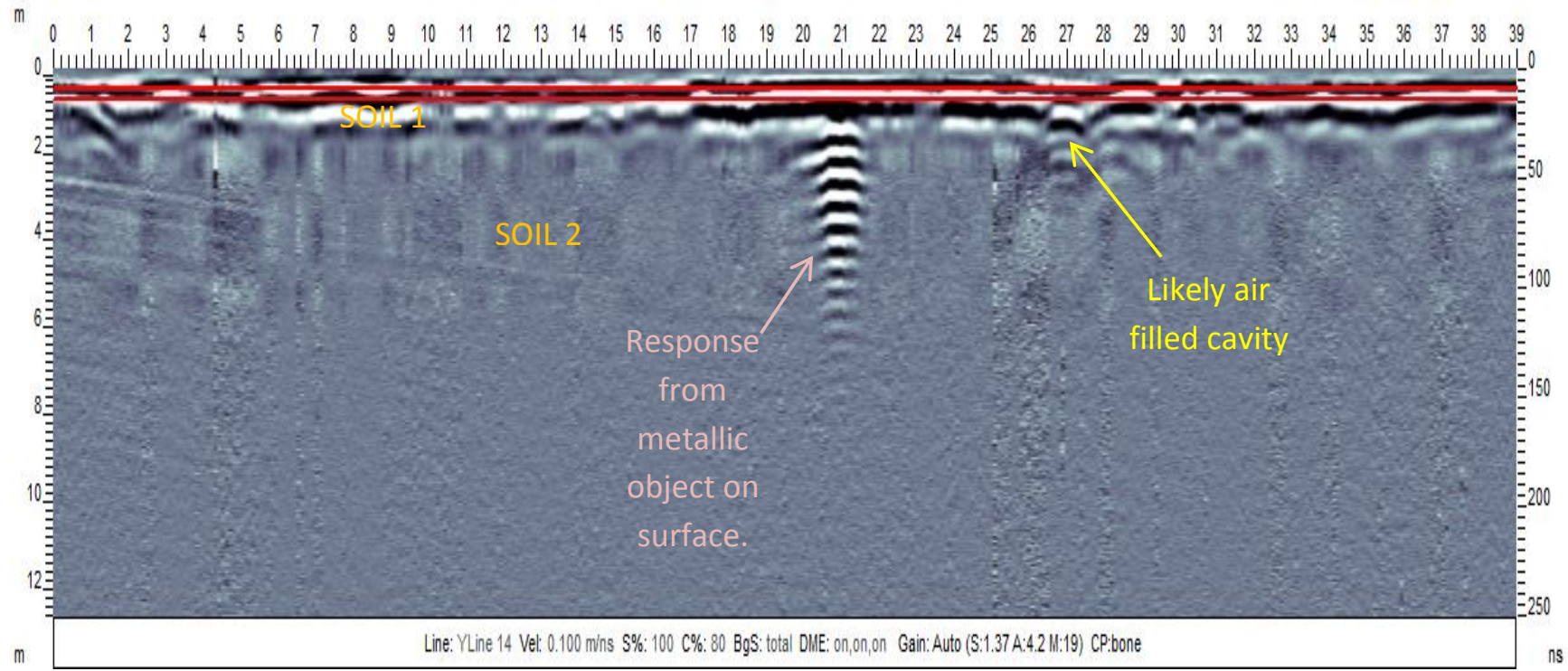

Figure 33: Vertical section of profile line 14 with slice lines at 0.25 to $0.50 \mathrm{~m}$

Figure 33 illustrates vertical section through profile line 14 with slice lines at 0.25 to $0.50 \mathrm{~m}$. A very ringy multiple response to a metallic object is observed at $21 \mathrm{~m}$ along the line. Similar features and metallic response was also notes in line 13. 
Figure 34, illustrates profile line collected during calibration walk-through, the profile line shows a constant horizontal feature at approximately $4.75 \mathrm{~m}$ below the surface. This horizontal feature was interpreted as the Ground Water Table (G.W.T). The gradual sloping of the G.W.T between $(0$ and $10 \mathrm{~m})$ along the profile line is due to the fact that, the surface is gradually sloping downwards there the radar pulses have a shorter travel time hence less depth. If a GPS system was used during data collection the software could perform a topographic correction which would flatten the G.W.T and contour the surface.

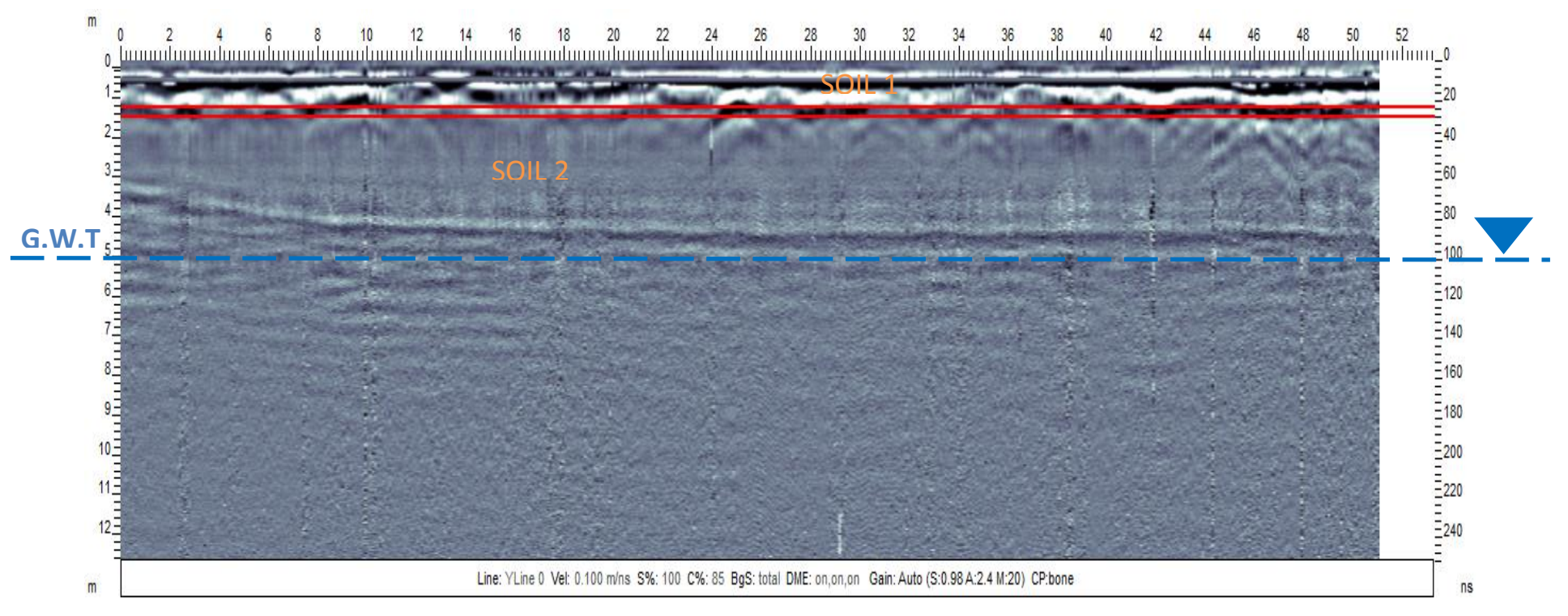

Figure 34: Section of profile collected during random walk through 


\subsubsection{Results Grid 4}

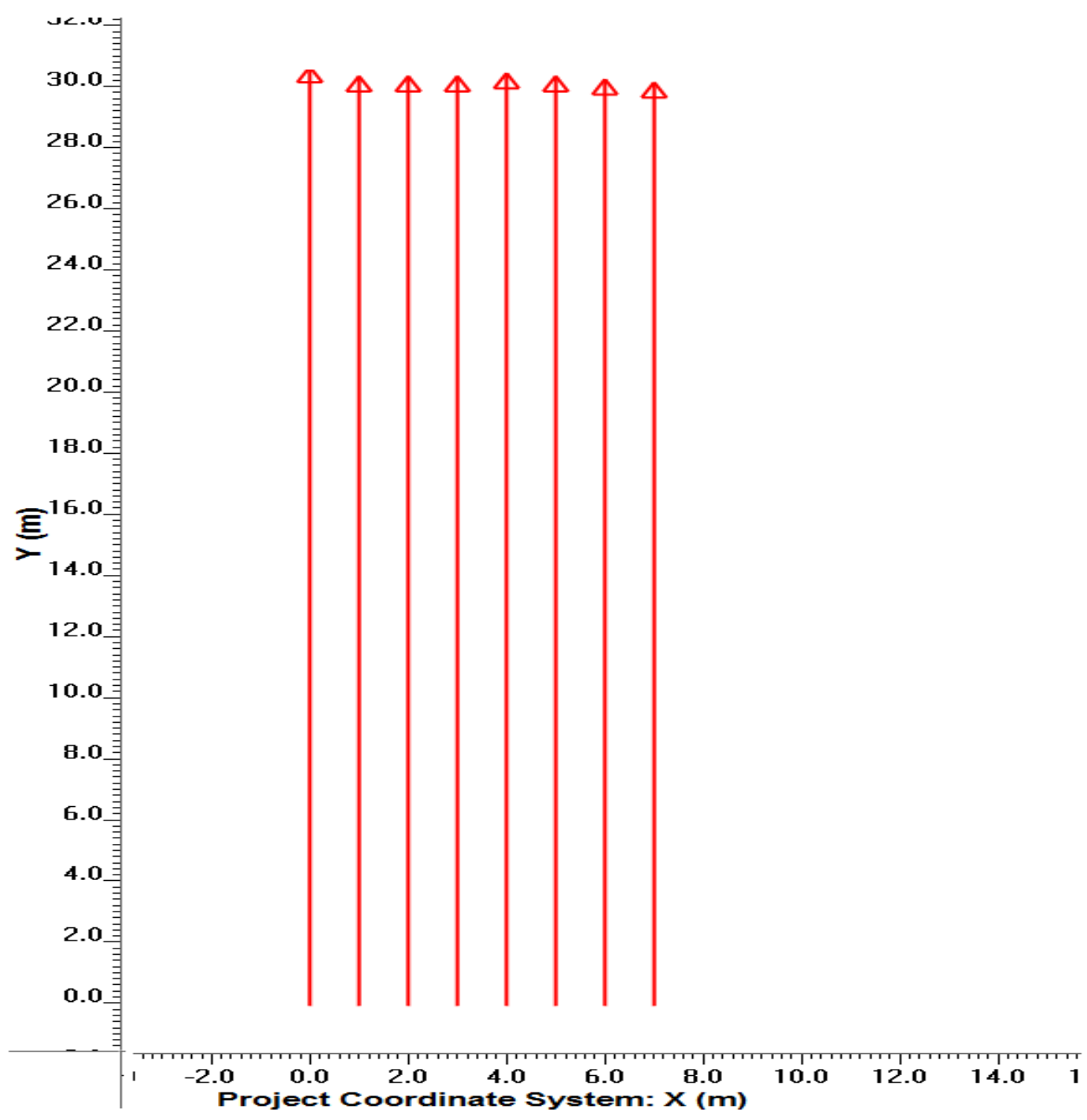

Figure 35: Illustrating profile lines collected in grid 4 . 


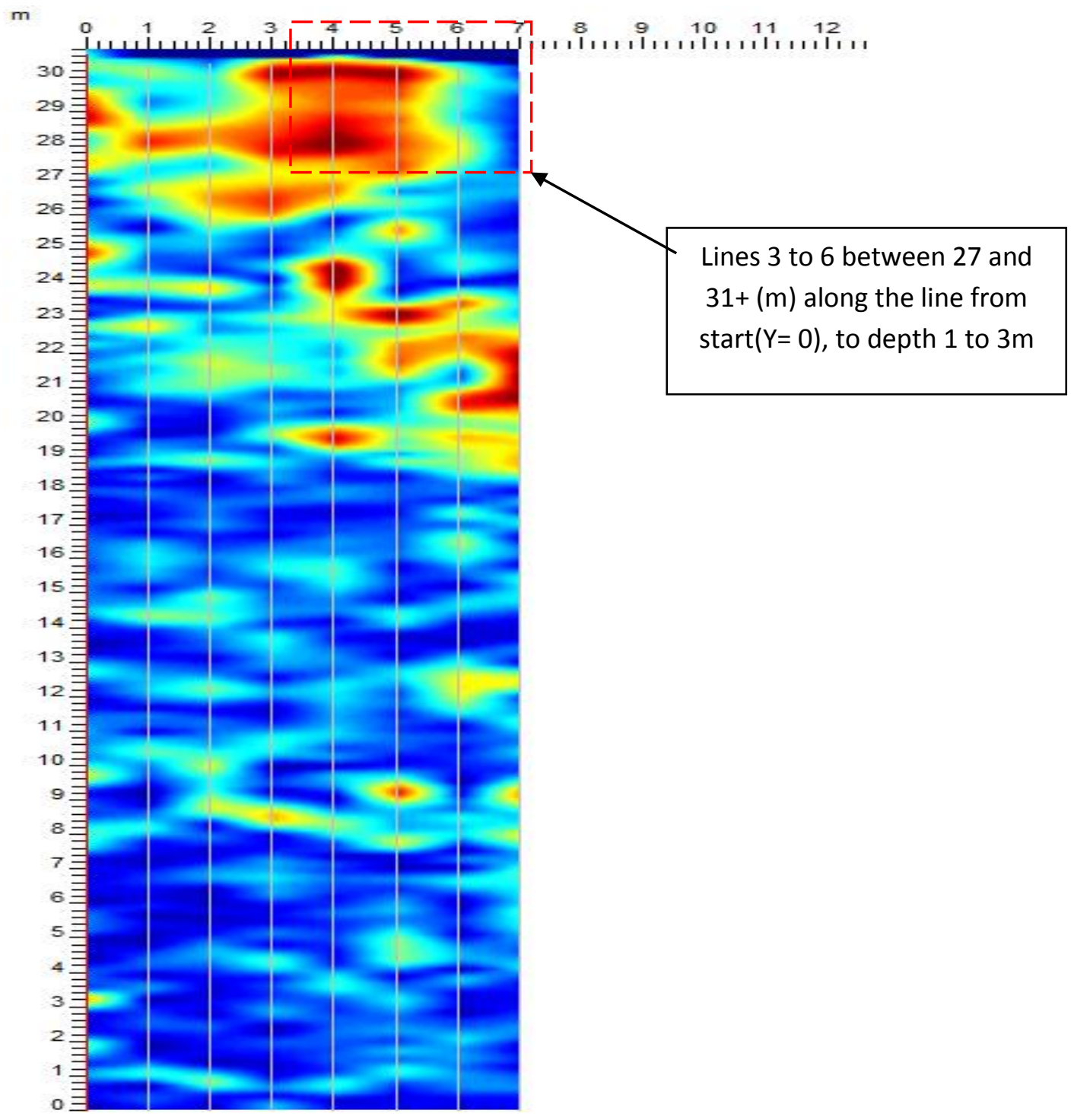

Figure 36: Horizontal Slice across Grid 4 at $0.50-0.75 \mathrm{~m}$ depth

Figure 36 illustrate, horizontal slice across Grid 4 at 0.50-0.75 depth, with the main area of concern highlighted in red dotted lines. 


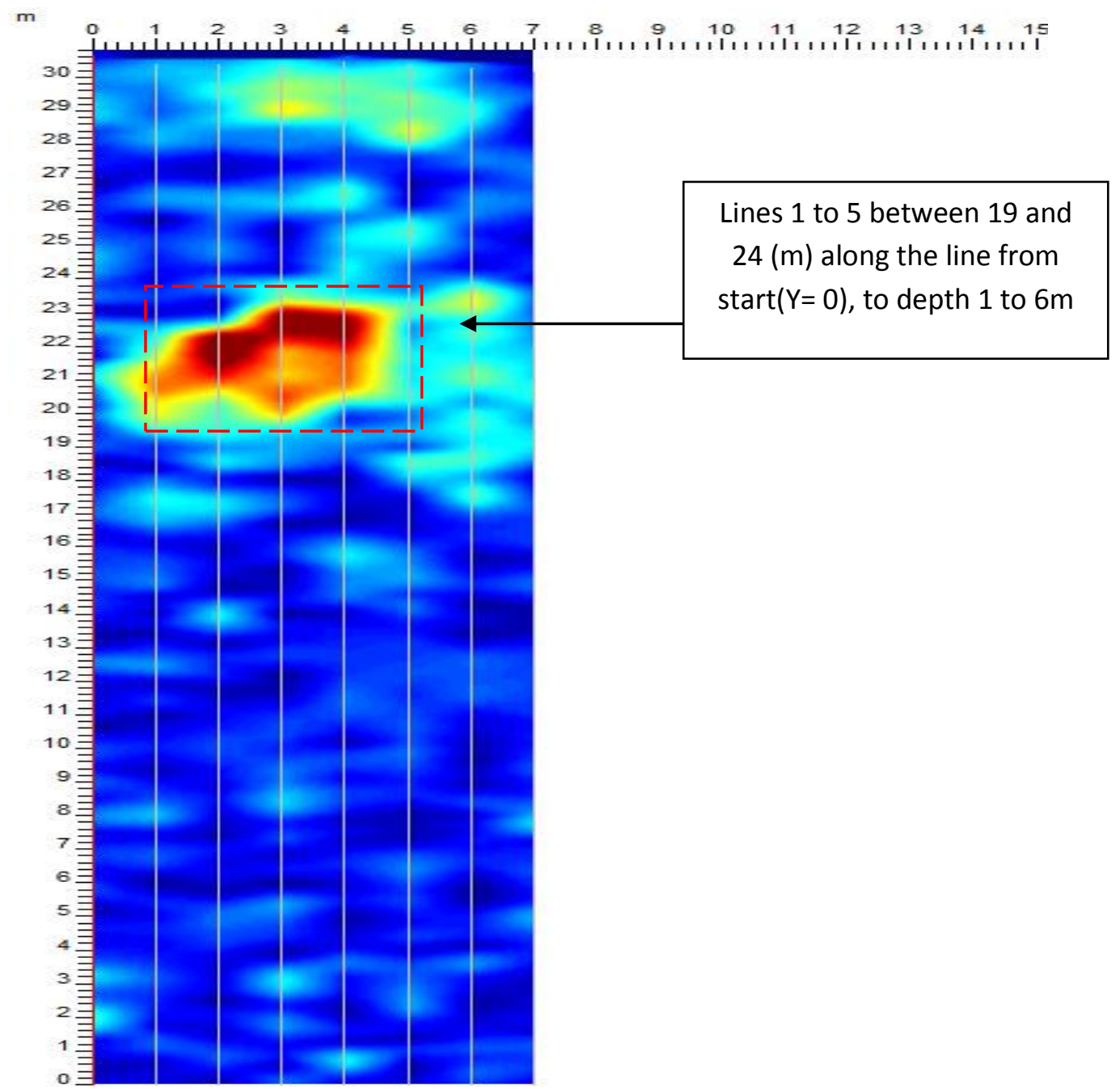

Figure 37: Horizontal Slice across Grid 4 at 1.25- 1.50m depth

Figure 37 illustrates, horizontal slice across Grid 4 at 1.25-1.50m depth, with the main area of concern highlighted in red dotted lines. 


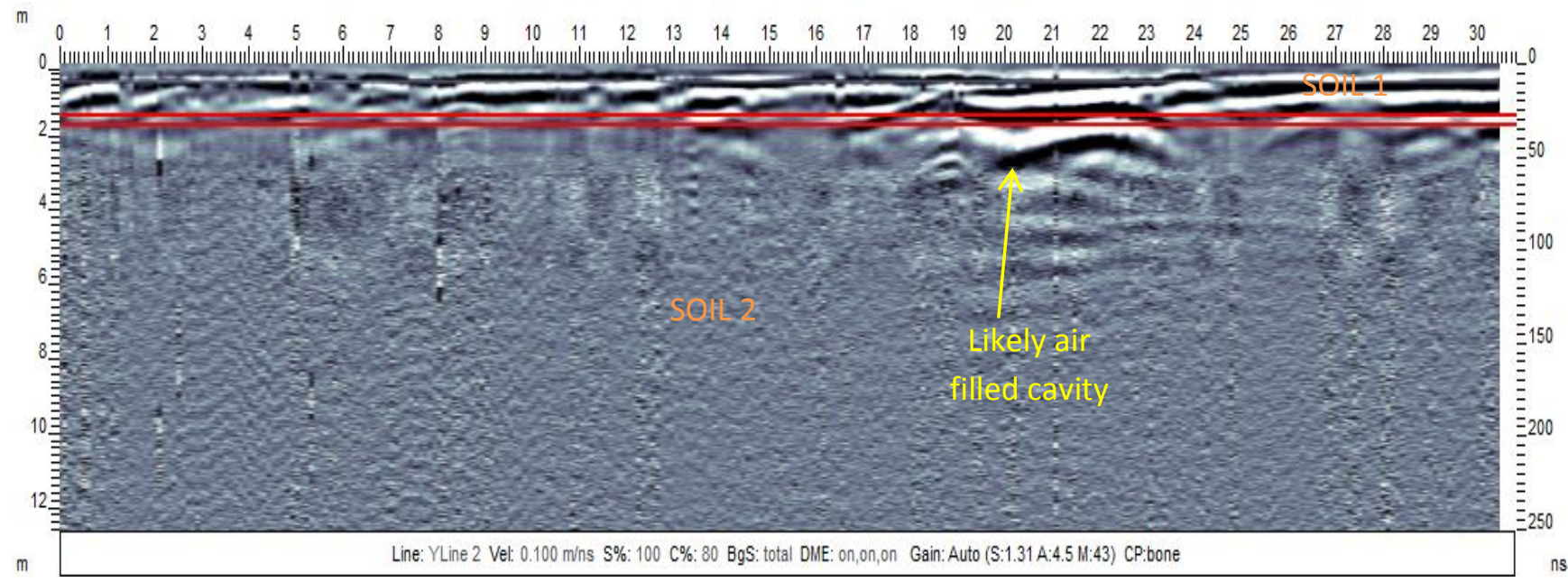

Figure 38: vertical section of profile line 2 from grid 4

Figure 38, shows processed vertical section of profile line 2 from grid 4 with slice lines at 1.50$1.75 \mathrm{~m}$ deep, indicating target between 19 and $24 \mathrm{~m}$. 


\subsubsection{Results Grid 5}

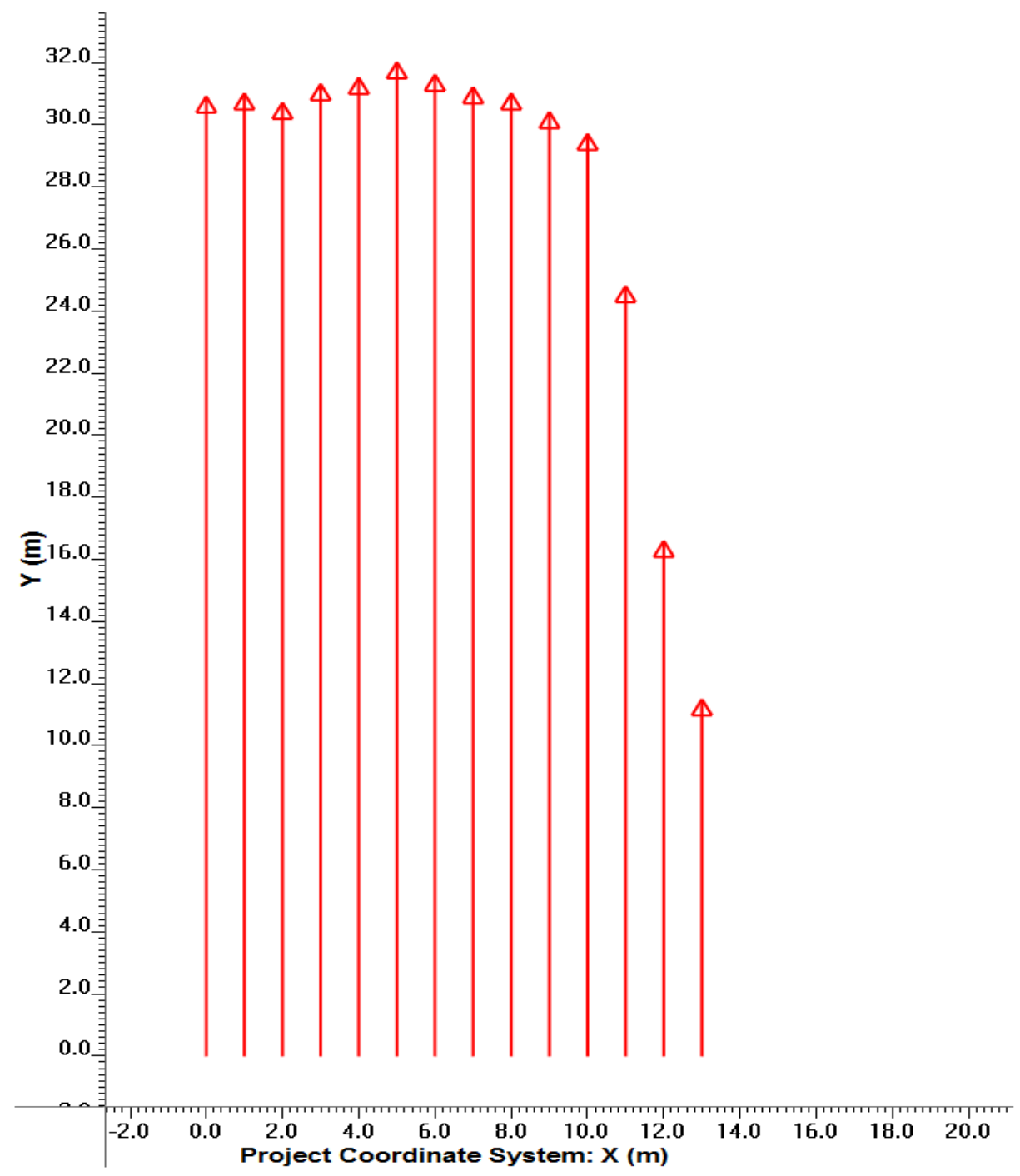

Figure 39: Illustrating profile lines collected in grid 5 


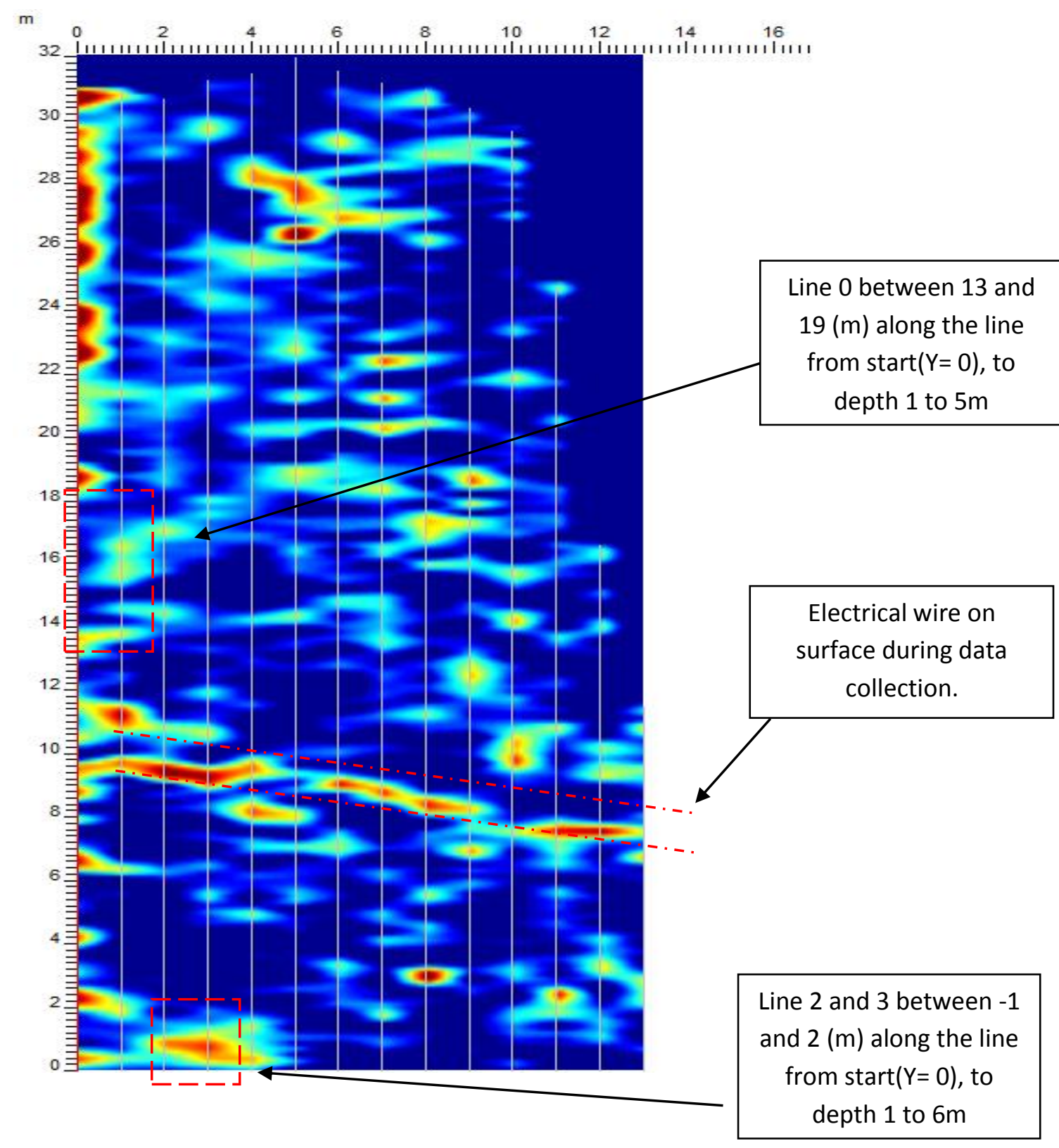

Figure 40: Horizontal slice across Grid 4 at 3.25-3.50m depth

Figure 40 illustrates, horizontal slice across Grid 4 at 3.25-3.50m depth, with the main area of concern highlighted in red dotted lines. 


\subsubsection{Summary of Results}

Figure 41, illustrates, the areas across the investigated site to find likely targets and anomalies, such as an air-filled cavity or loosely deposited soils. The figure is a schematic representation of the combined results from grids 0, 1, 4, and 5 shown in Figure 26, Figure 31, Figure 36, and Figure 40 respectively. Due to shallow depths of these targets they were excavated and filled with high strength concrete before starting foundation works.

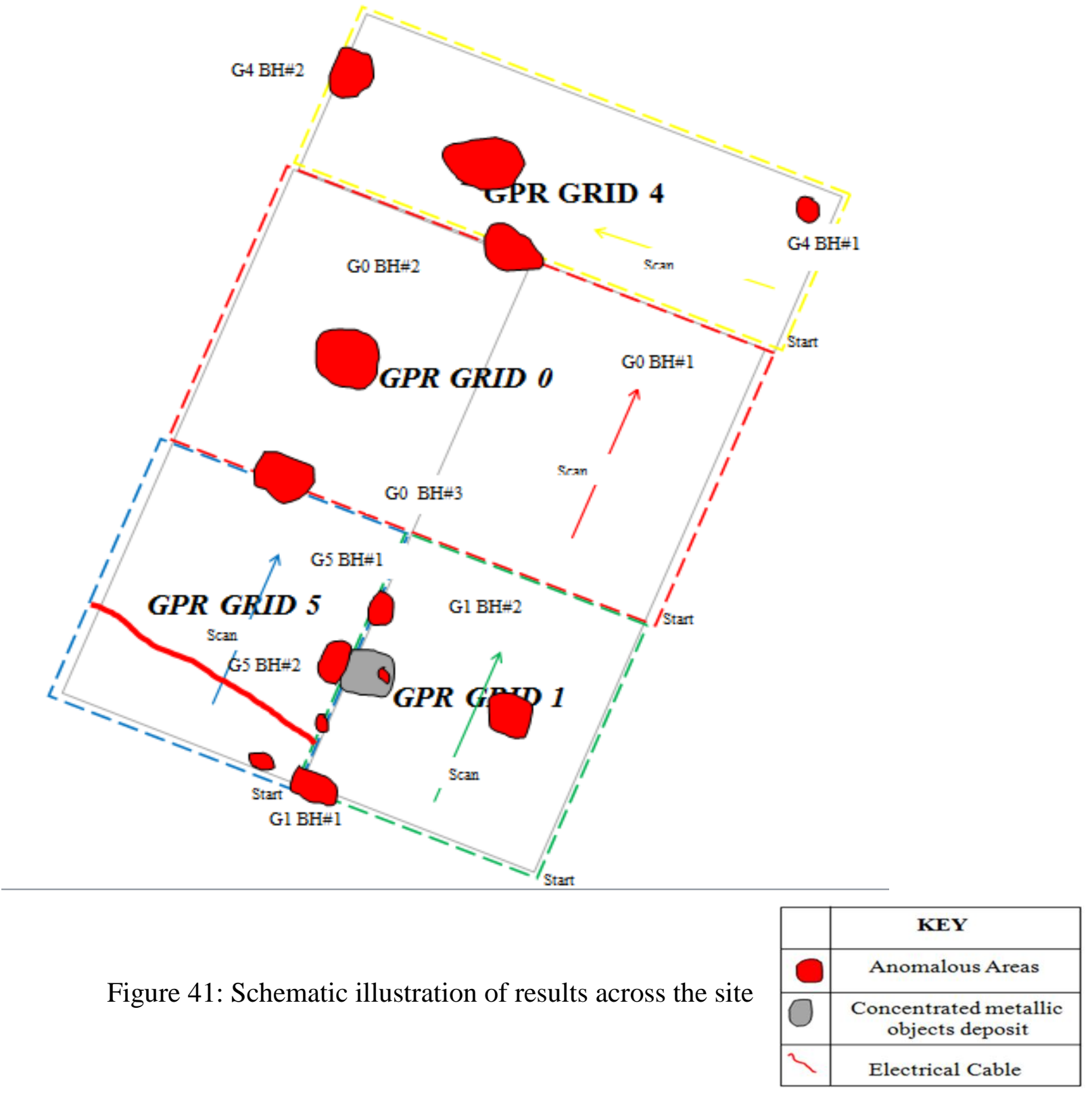




\section{CHAPTER 5}

\section{CONCLUSION, RECOMMEDATION AND LIMITATIONS}

\subsection{Conclusion and Recommendation}

This project has highlighted GPR has a viable tool to be used alone or in conjunction with traditional methods of geotechnical investigatory procedures to gain supplementary information about the investigated site hence reducing the risks and uncertainties that exist in the geotechnical data collection process. GPR was also able to differentiate the various soil layers at depth to aid soil profile presumptions between boreholes and test areas. This can be attributed to GPR continuous signal and reflection profiling data acquisition process and accuracy in detecting changes in electrical properties. Correlating GPR data and SPT or CPT data can increase design competence, reduce cost associated with gathering more information by traditional methods. GPR used in geotechnical investigation can also provide a means to reduce subjective interpretation by less experience engineers since more data would be available for review

It has been illustrated that GPR can produce qualitative and quantitative data due to its ability to identify varying dielectric and conductivity properties of different soil types. Vast information is stored in the transmitted and reflected wave as it passes through the soil medium which can be quantified to relate to physical and mechanical properties of the soil void ratio, moisture and angle of internal friction for sandy soils where illustrated in this project. 


\subsection{Limitations of GPR}

1. Gradual changes from materials having similar dielectric properties will be difficult to be detect by GPR detailed in section 2.4.1

2. GPR cannot conclusively specify the composition of the materials/soil without correlation and observation. Interpretations and estimates of the soil types can be made from GPR reflections by experience individuals. Confirmation of soil types can be made by traditional borings. Boreholes can be correlated with GPR data to reduce the amount of borehole normally required.

3. Conductivity of the material is also a limitation. Salt water and wet clays can partially or totally absorb GPR signals. The absorbed signals can prevent GPR being feasible for such jobs since limited penetration will be achieved in these conditions especially if the clay located on top. Clays at lower depth can be differentiated from other surrounding soils since the clay will absorb the GPR transmitted pulse.

4. Scattering of reflected waves inconsistent soils can causes micro internal reflections, these reflections does not have enough energy to return to the receiver on the surface. Hence a loss of sign will occur.

5. High energy reflections from highly contrast boundaries, in this case all or most of energy at the soil interface boundary is reflected, hence small amount of transmitted energy gets refracted into the second medium. This will result in low penetration or small reflections from the second medium. 


\subsection{Future Study}

Upon completion of this project it has been realized that GPR has gained much recognition as a fast, accurate and reliable tool with many applications in geotechnical engineering and civil engineering. These attributes are due to increase technology and data processing found in modern GPR systems. Further studies into extracting quantifiable information from GPR data would be recommended for future studies. Particularly information relating to the determination of the composition of the soil, this would provide greater understanding of the sub-surface soils, and further reduce subjective soil profile presumptions by engineers. Presently GPR data is correlated with SPT or CPT data to determine the composition of the materials providing the reflections. 


\section{References}

Anna, P. (2003). Ground Penetrating Radar Principles Application and Procedure.

Annan, A. P. (2001). GPR - History, Trends \& Future Developments. 1-2.

Baker, J. (2007). Stratigraphic Analyses using GPR. Colorado: The Geological Society of America.

Carling, B. (2016). Ground-penetrating radar stratigraphy and dynamics of megaflood gravel dunes. Geological Society.

Clemeña, G.G. (2004)."Short-Pulse Radar Methods." In: Handbook on Nondestructive Testing of Concrete, Malhotra, V.M and Carino, N.J., ed., 2nd Ed., ASTM \& CRC.

GeoSense. (n.d.). Retrieved 07 6, 2017, from Geo Sense: http://geo-

sense.com/index.php/methods/ground-penetrating-radar-gpr-surveys/

Gregory B. Baecher, J. T. (2003). Reliablity and Statistics in Geotechnical Engineering.

GSSI. (2001). GSSI. Retrieved 06 08, 2017, from GSSI:

http://www.geophysical.com/geology.htm

Huisman, J.A., C. Sperl, W. Bouten, and J.M. Verstraten. (2001). Soil water content measurements at different scales: Accuracy of time domain reflectometry and ground-penetrating radar.

Interreg. (n.d.). Recommendations for guidelines for the use of GPR in site investigation.

J.A. Huisman, S. H. (n.d.). Measuring Soil Water Content with Ground Penetrating Radar.

Jangda, C. (2014, November 2). Retrieved March 19, 2017, from Ground Pentrating Radar

(GPR): http://chetanjangda007.blogspot.ca/2014/11/ground-penetrating-radar-gpr.html

Jol, H. M. (2009). Ground Pentrating Radar Theory and Application. Amsterdam: Elsevier.

Mitchell, J. (1993). Fundamentals of soil behavior 2nd Edition. John Wiley and Sons.

NASA. (2012, November 10). Retrieved February 1, 2017, from NASA:

https://www.nasa.gov/directorates/heo/scan/communications/outreach/funfacts/txt_band_designa tors.html

Pilon, J. (1992). Ground Penetrating Radar. Canada: Canada Communication Group.

Robert E. Kayen, 1. W. (1999). High-Resolution Crosshole Radar Tomography:Application to Liquefaction-Induced Changes. 
Sensors and Software, S. (2014). Ground Pentrating Radar: Principles, Procedures and Application. Mississauaga.

Terzaghi, P. M. (1996). Soil Mechanics in Engineering Practice. New York: Library of Congress..

Tillard, S., and J.-C. Dubois. (1995). Analysis of GPR data: Wave propagation velocity determination. J. Appl

Yelf, R. J. (2006). Application of Ground Penetrating Radar to Civil and Geotechnical Engineering. 